TRANSACTIONS OF THE

AMERICAN MATHEMATICAL SOCIETY

Volume 359, Number 7, July 2007, Pages 3005-3024

S 0002-9947(07)04158-X

Article electronically published on February 21, 2007

\title{
THE $G$-STABLE PIECES OF THE WONDERFUL COMPACTIFICATION
}

\author{
XUHUA HE
}

\begin{abstract}
Let $G$ be a connected, simple algebraic group over an algebraically closed field. There is a partition of the wonderful compactification $\bar{G}$ of $G$ into finite many $G$-stable pieces, which was introduced by Lusztig. In this paper, we will investigate the closure of any $G$-stable piece in $\bar{G}$. We will show that the closure is a disjoint union of some $G$-stable pieces, which was first conjectured by Lusztig. We will also prove the existence of cellular decomposition if the closure contains finitely many $G$-orbits.
\end{abstract}

\section{INTRODUCTION}

An adjoint semi-simple group $G$ has a "wonderful" compactification $\bar{G}$, introduced by De Concini and Procesi in [DP. The variety $\bar{G}$ is a smooth variety with $G \times G$ action. Denote by $G_{\text {diag }}$ the image of the diagonal embedding of $G$ into $G \times G$. The $G_{d i a g}$-orbits of $\bar{G}$ were studied by Lusztig in [L4]. He introduced a partition of $\bar{G}$ into finitely many $G$-stable pieces. The $G$-orbits on each piece can be described explicitly. Based on the partition, he established the theory of "parabolic character sheaves" on $\bar{G}$.

The main results of this paper concern the closure of the $G$-stable pieces. The closure of each piece is a union of some other pieces, and if the closure contains finitely many $G$-orbits, then it admits a cellular decomposition. I believe that our results are necessary ingredients for establishing the (conjectural) Kazhdan-Lusztig theory on the "Parabolic Character Sheaves" on $\bar{G}$.

We now review the content of this paper in more detail.

In section 1, we recall the definition of $G$-stable pieces in [L4] and establish some basic results. The pieces are indexed by the pairs $\mathcal{I}=\{(J, w)\}$, where $J$ is a subset of the simple roots and $w$ is an element of the Weyl group $W$, which has minimal length in the coset $w W_{J}$. One interesting result is that any $G$-stable piece is the minimal $G$-stable subset that contains a particular $B \times B$-orbit, where $B$ is the Borel subgroup. The closure of any $B \times B$-orbit in $\bar{G}$ was studied by Springer in $[\mathrm{S}$. Based on his result and the relations between $G$-stable pieces and $B \times B$-orbits, we are able to investigate the closure of the $G$-stable pieces.

In section 2, we recall the definition of the "wonderful" compactification and introduce "compactification through the fibres", a technique tool that will be used to prove the existence of cellular decomposition. In section 3, we describe a partial order on $\mathcal{I}$, which is the partial order that corresponds to the closure relation of the

Received by the editors March 4, 2005

2000 Mathematics Subject Classification. Primary 20G15, 14L30.

(C)2007 American Mathematical Society Reverts to public domain 28 years from publication 
$G$-stable piece, as we will see in section 4 . In section 4, we also discuss the closure of any $G$-stable piece that appears in [L3].

In section 5, we discuss the existence of cellular decomposition. Each piece does not have a cellular decomposition. However, a union of certain pieces has a cellular decomposition. (This is motivated by Springer in $[\underline{S}$, in which he showed that a union of certain $B \times B$-orbits is isomorphic to an affine space.) In fact, if the closure contains finitely many $G$-orbits, then it has a cellular decomposition.

The methods work for an arbitrary connected component of a disconnected algebraic group with identity component $G$. The results for that component is just a "twisted" version of the results for $G$ itself.

\section{The $G$-STABle PIECES}

1.1. In the sequel $G$ is a connected, semi-simple algebraic group of adjoint type over an algebraically closed field. Let $B$ be a Borel subgroup of $G, B^{-}$be the opposite Borel subgroup and $T=B \cap B^{-}$. Let $\left(\alpha_{i}\right)_{i \in I}$ be the set of simple roots. For $i \in I$, we denote by $s_{i}$ the corresponding simple reflection. For any element $w$ in the Weyl group $W=N(T) / T$, we will choose a representative $\dot{w}$ in $N(T)$ in the same way as in [L1, 1.1].

For $J \subset I$, let $P_{J} \supset B$ be the standard parabolic subgroup defined by $J$ and $P_{J}^{-} \supset B^{-}$be the opposite of $P_{J}$. Set $L_{J}=P_{J} \cap P_{J}^{-}$. Then $L_{J}$ is a Levi subgroup of $P_{J}$ and $P_{J}^{-}$. We denote by $\Phi_{J}$ the set of roots that are a linear combination of $\left\{\left(a_{j}\right)_{j \in J}\right\}$. Let $Z_{J}$ be the center of $L_{J}$ and $G_{J}=L_{J} / Z_{J}$ be its adjoint group. We denote by $\pi_{P_{J}}$ (resp. $\pi_{P_{J}^{-}}$) the projection of $P_{J}$ (resp. $P_{J}^{-}$) onto $G_{J}$.

For any $J \subset I$, let $\mathcal{P}_{J}$ be the set of parabolic subgroups conjugate to $P_{J}$. We will write $\mathcal{B}$ for $\mathcal{P}_{\varnothing}$.

For any subset $J$ of $I$, let $W_{J}$ be the subgroup of $W$ generated by $\left\{s_{j} \mid j \in J\right\}$ and $W^{J}$ (resp. ${ }^{J} W$ ) be the set of minimal length coset representatives of $W / W_{J}$ (resp. $W_{J} \backslash W$ ). Let $w_{0}^{J}$ be the unique element of maximal length in $W_{J}$. (We will simply write $w_{0}^{I}$ as $w_{0}$.) For $J, K \subset I$, we write ${ }^{J} W^{K}$ for ${ }^{J} W \cap W^{K}$.

For $w \in W$, we denote by $\operatorname{supp}(w) \subset I$ the set of simple roots whose associated simple reflections occur in some (or equivalently, any) reduced decomposition of $w$.

For $J, K \subset I, P \in \mathcal{P}_{J}, Q \in \mathcal{P}_{K}$ and $u \in{ }^{J} W^{K}$, we write $\operatorname{pos}(P, Q)=u$ if there exists $g \in G$, such that ${ }^{g} P=P_{J}$ and ${ }^{g} Q={ }^{\dot{u}} P_{K}$.

For any parabolic subgroup $P$, we denote by $U_{P}$ its unipotent radical. We will simply write $U$ for $U_{B}$ and $U^{-}$for $U_{B^{-}}$. For $J \subset I$, set $U_{J}=U \cap L_{J}$ and $U_{J}^{-}=U^{-} \cap L_{J}$.

For any closed subgroup $H$ of $G$, we denote by $H_{\text {diag }}$ the image of the diagonal embedding of $H$ in $G \times G$. For any subgroup $H$ and $g \in G$, we write ${ }^{g} H$ for $g H g^{-1}$. For any finite set $A$, we write $|A|$ for the cardinal of $X$.

1.2. Let $\hat{G}$ be a possibly disconnected reductive algebraic group over an algebraically closed field with identity component $G$. Let $G^{1}$ be a fixed connected component of $\hat{G}$. There exists an isomorphism $\delta: W \rightarrow W$ such that $\delta(I)=I$ and ${ }^{g} P \in \mathcal{P}_{\delta(J)}$ for $g \in G^{1}$ and $P \in \mathcal{P}_{J}$. There also exists $g_{0} \in G^{1}$ such that $g_{0}$ normalizes $T$ and $B$. Moreover, $g_{0}$ can be chosen in such a way that ${ }^{g_{0}} L_{J}=L_{\delta(J)}$ for $J \subset I$. We will fix such $g_{0}$ in the rest of this paper.

In particular, if $G^{1}=G$, then $\delta=i d$, where $i d$ is the identity map. In this case, we choose $g_{0}$ to be the unit element 1 of $G$. 
1.3. We will follow the set-up of [L4].

Let $J, J^{\prime} \subset I$ and $y \in{ }^{J^{\prime}} W^{J}$ be such that $\operatorname{Ad}(y) \delta(J)=J^{\prime}$. For $P \in \mathcal{P}_{J}, P^{\prime} \in \mathcal{P}_{J^{\prime}}$, define $A_{y}\left(P, P^{\prime}\right)=\left\{g \in G^{1} \mid \operatorname{pos}\left(P^{\prime},{ }^{g} P\right)=y\right\}$. Define

$$
\tilde{Z}_{J, y, \delta}=\left\{\left(P, P^{\prime}, \gamma\right) \mid P \in \mathcal{P}_{J}, P^{\prime} \in \mathcal{P}_{J^{\prime}}, \gamma \in U_{P^{\prime}} \backslash A_{y}\left(P, P^{\prime}\right) / U_{P}\right\}
$$

with $G \times G$ action defined by $\left(g_{1}, g_{2}\right)\left(P, P^{\prime}, \gamma\right)=\left({ }^{g_{2}} P,{ }^{g_{1}} P^{\prime}, g_{1} \gamma g_{2}^{-1}\right)$.

By [L4, 8.9], $A_{y}\left(P, P^{\prime}\right)$ is a single $P^{\prime}, P$ double coset. Thus $G \times G$ acts transitively on $Z_{J, y, \delta}$.

Let $z=\left(P, P^{\prime}, \gamma\right) \in \tilde{Z}_{J, y, \delta}$. Then there exists $g \in \gamma$ such that ${ }^{g} P$ contains some Levi of $P \cap P^{\prime}$. Now set $P_{1}=g^{-1}\left({ }^{g} P\right)^{\left(P^{\prime P}\right)} g, P_{1}^{\prime}=P^{P^{\prime}}$. Define

$$
\alpha\left(P, P^{\prime}, \gamma\right)=\left(P_{1}, P_{1}^{\prime}, U_{P_{1}^{\prime}} g U_{P_{1}}\right) .
$$

By [L4, 8.11], the map $\alpha$ doesn't depend on the choice of $g$.

To $z=\left(P, P^{\prime}, \gamma\right) \in \tilde{Z}_{J, y, \delta}$, we associate a sequence $\left(J_{k}, J_{k}^{\prime}, u_{k}, y_{k}, P_{k}, P_{k}^{\prime}, \gamma_{k}\right)_{k \geqslant 0}$ with $J_{k}, J_{k}^{\prime} \subset I, u_{k} \in W, y_{k} \in J_{k}^{\prime} W^{\delta\left(J_{k}\right)}, \operatorname{Ad}\left(y_{k}\right) \delta\left(J_{k}\right)=J_{k}^{\prime}, P_{k} \in \mathcal{P}_{J_{k}}, P_{k}^{\prime} \in$ $\mathcal{P}_{J_{k}^{\prime}}, \gamma_{k}=U_{P_{k}^{\prime}} g U_{P_{k}}$ for some $g \in G$ that satisfies $\operatorname{pos}\left(P_{k}^{\prime},{ }^{g} P_{k}\right)=y_{k}$. The sequence is defined as follows:

$$
P_{0}=P, P_{0}^{\prime}=P^{\prime}, \gamma_{0}=\gamma, J_{0}=J, J_{0}^{\prime}=J^{\prime}, u_{0}=\operatorname{pos}\left(P_{0}^{\prime}, P_{0}\right), y_{0}=y .
$$

Assume that $k \geqslant 1$, that $P_{m}, P_{m}^{\prime}, \gamma_{m}, J_{m}, J_{m}^{\prime}, u_{m}, y_{m}$ are already defined for $m<k$ and that $u_{m}=\operatorname{pos}\left(P_{m}^{\prime}, P_{m}\right), P_{m} \in \mathcal{P}_{J_{m}}, P_{m}^{\prime} \in \mathcal{P}_{J_{m}^{\prime}}$ for $m<k$. Let

$$
\begin{gathered}
J_{k}=J_{k-1} \cap \delta^{-1} \operatorname{Ad}\left(y_{k-1}^{-1} u_{k-1}\right) J_{k-1}, J_{k}^{\prime}=J_{k-1} \cap \operatorname{Ad}\left(u_{k-1}^{-1} y_{k-1}\right) \delta\left(J_{k-1}\right), \\
\left.\left(P_{k}, P_{k}^{\prime}, \gamma_{k}\right)=\alpha\left(P_{k-1}, P_{k-1}^{\prime}, \gamma_{k-1}\right) \in \tilde{Z}_{J_{k}, y_{k}, \delta}(\text { see [L4 }, 8.10]\right), \\
u_{k}=\operatorname{pos}\left(P_{k}^{\prime}, P_{k}\right), y_{k}=u_{k-1}^{-1} y_{k-1}, \gamma_{k}=U_{P_{k}^{\prime}} g_{k-1} U_{P_{k}} .
\end{gathered}
$$

It is known that the sequence is well defined. Moreover, for sufficiently large $n$, we have that $J_{n}=J_{n}^{\prime}=J_{n+1}=J_{n+1}^{\prime}=\cdots=J_{\infty}, u_{n}=u_{n+1}=\cdots=1$, $y_{n}=y_{n+1}=\cdots=y_{\infty}, P_{n}=P_{n+1}=\cdots=P_{\infty}, P_{n}^{\prime}=P_{n+1}^{\prime}=\cdots=P_{\infty}^{\prime}$ and $\gamma_{n}=\gamma_{n+1}=\cdots=\gamma_{\infty}$. Now we set $\beta(z)=y_{\infty}$. Then we have that $\beta(z) \in W^{\delta(J)}$. By [L4, 8.18] and [L3, 2.5], the sequence $\left(J_{n}, J_{n}^{\prime}, u_{n}, y_{n}\right)_{n \geqslant 0}$ is uniquely determined by $\beta(z)$ and $y$.

For $w \in W^{\delta(J)}$, set

$$
\tilde{Z}_{J, y, \delta}^{w}=\left\{z \in \tilde{Z}_{J, y, \delta} \mid \beta(z)=w\right\} .
$$

Then $\left(\tilde{Z}_{J, y, \delta}^{w}\right)_{w \in W^{\delta(J)}}$ is a partition of $\tilde{Z}_{J, y, \delta}$ into locally closed $G$-stable subvarieties. We call $\left(\tilde{Z}_{J, y, \delta}^{w}\right)_{w \in W^{\delta(J)}}$ the $G$-stable pieces of $\tilde{Z}_{J, y, \delta}$. For $w \in W^{\delta(J)}$, let $\left(J_{n}, J_{n}^{\prime}, u_{n}, y_{n}\right)_{n \geqslant 0}$ be the sequence determined by $w$ and $y$. The restriction of the map $\alpha$ on $\tilde{Z}_{J, y, \delta}^{w}$ is a $G$-equivariant morphism from $\tilde{Z}_{J, y, \delta}^{w}$ onto $\tilde{Z}_{J_{1}, y_{1}, \delta}^{w}$. We also denote this morphism by $\alpha$. It is known that $\alpha$ induces a bijection from the set of $G$-orbits on $\tilde{Z}_{J, y, \delta}^{w}$ to the set of $G$-orbits on $\tilde{Z}_{J_{1}, y_{1}, \delta}^{w}$.

We have a consequence $\tilde{Z}_{J, y, \delta}^{w} \stackrel{\alpha}{\rightarrow} \tilde{Z}_{J_{1}, y_{1}, \delta}^{w} \stackrel{\alpha}{\rightarrow} \tilde{Z}_{J_{2}, y_{2}, \delta}^{w} \stackrel{\alpha}{\rightarrow} \cdots$. For sufficiently large $n, \vartheta=\alpha^{n}: \tilde{Z}_{J, y, \delta}^{w} \rightarrow \tilde{Z}_{J_{\infty}, w, \delta}^{w}$ is independent of the choice of $n$ and is a $G$-equivariant morphism. Moreover, $\vartheta$ induces a bijection from the set of $G$-orbits on $\tilde{Z}_{J, y, \delta}^{w}$ to the set of $G$-orbits on $\tilde{Z}_{J \infty, w, \delta}^{w}$.

In the rest of this section, we will fix $J, y, \delta, w$ and $J_{\infty}$. First, we will give an explicit description of $J_{\infty}$ in terms of $J, \delta$ and $w$. 
Lemma 1.4. Keep the notion of section 1.3. Then

$$
J_{\infty}=\max \{K \subset J \mid \operatorname{Ad}(w) \delta(K)=K\} .
$$

Proof. Set $v=y_{1} w^{-1}$. By [ㅍ, 2.2], $v \in W_{J}$. Now $J_{1}=J \cap \delta^{-1} \operatorname{Ad}\left(y_{1}^{-1}\right) J$. Thus $\Phi_{\delta\left(J_{1}\right)} \subset \operatorname{Ad}\left(y_{1}^{-1}\right) \Phi_{J}=\operatorname{Ad}\left(w^{-1}\right) \operatorname{Ad}\left(v^{-1}\right) \Phi_{J}=\operatorname{Ad}\left(w^{-1}\right) \Phi_{J}$.

Let $i \in J$. Assume that $\alpha_{\delta(i)} \in \operatorname{Ad}\left(y_{1}^{-1}\right) \Phi_{J}$. Then $\alpha_{\delta(i)}=\operatorname{Ad}\left(y_{1}^{-1}\right) \alpha=$ $\operatorname{Ad}\left(y^{-1}\right) \operatorname{Ad}\left(u_{0}\right) \alpha$ for some $\alpha \in \Phi_{J}$. Then $\alpha_{\operatorname{Ad}(y) \delta(i)}=\operatorname{Ad}\left(u_{0}\right) \alpha$. Note that $\alpha_{\operatorname{Ad}(y) \delta(i)}$ is a simple root and $u_{0} \in W^{J}$. Then $\alpha=\alpha_{j}$ for some $j \in J$. Hence $i=\delta^{-1} \operatorname{Ad}\left(y_{1}^{-1}\right) j$. Therefore, $i \in J \cap \delta^{-1} \operatorname{Ad}\left(y_{1}^{-1}\right) J=J_{1}$. So

$$
J_{1}=\max \left\{K \subset J \mid \Phi_{\delta(K)} \subset \operatorname{Ad}\left(w^{-1}\right) \Phi_{J}\right\} .
$$

Set $J_{\infty}^{\prime}=\max \{K \subset J \mid \operatorname{Ad}(w) \delta(K)=K\}$. Then $J_{\infty}^{\prime} \subset J$. Moreover, $\Phi_{\delta\left(J_{\infty}^{\prime}\right)}=$ $\operatorname{Ad}\left(w^{-1}\right) \Phi_{J_{\infty}^{\prime}} \subset \operatorname{Ad}\left(w^{-1}\right) \Phi_{J}$. Thus $J_{\infty}^{\prime} \subset J_{1}$. We can show by induction that $J_{\infty}^{\prime} \subset J_{n}$ for all $n$. Thus $J_{\infty}^{\prime} \subset J_{\infty}$. By the definition, $J_{\infty}=J_{\infty} \cap \delta^{-1} \operatorname{Ad}\left(w^{-1}\right) J_{\infty}$. Thus $\operatorname{Ad}(w) \delta\left(J_{\infty}\right)=J_{\infty}$. So $J_{\infty}=J_{\infty}^{\prime}$. The lemma is proved.

1.5. Now set $\tilde{h}_{J, y, \delta}=\left(P_{J}, \dot{y}^{-1} P_{J^{\prime}}, U_{\dot{y}^{-1} P_{J^{\prime}}} g_{0} U_{P_{J}}\right) \in Z_{J, y, \delta}$. For $w \in W^{\delta(J)}$ and $v \in W$, set $[\widetilde{J, w, v}]_{y, \delta}=(B \times B)(\dot{w}, \dot{v}) \cdot \tilde{h}_{J, y, \delta}$. Then we have the following result.

Lemma 1.6. Keep the notion of section 1.3. Let $g \in P_{J_{1}}$. Set $z=(\dot{w}, g) \cdot \tilde{h}_{J, y, \delta}$ and $z^{\prime}=(\dot{w}, g) \cdot \tilde{h}_{J_{1}, y_{1}, \delta}$. Then $\alpha(z)=z^{\prime}$.

Proof. Set $P=P_{J}, P^{\prime}=\dot{w} \dot{y}^{-1} P_{J^{\prime}}, g_{1}=\dot{w} g_{0} g$ and $v=y_{1} w^{-1}$. Then $v \in W_{J}$.

By the proof of [H, 2.3], ${ }^{\dot{v}^{-1}} L_{J_{1}^{\prime}}$ is a Levi factor of $P \cap P^{\prime}$ and $P^{P^{\prime}}={ }^{\dot{v}^{-1}} P_{J_{1}^{\prime}}$, $\left(P^{\prime}\right)^{P}=\dot{w} \dot{y}^{-1} P_{\operatorname{Ad}(y) \delta\left(J_{1}\right)}$. Moreover,

$$
\dot{v}^{-1} L_{J_{1}^{\prime}}=\dot{w} \dot{y}_{1}^{-1} L_{J_{1}^{\prime}}={ }^{\dot{w}} L_{\delta\left(J_{1}\right)}={ }^{\dot{w} g_{0}} L_{J_{1}} \subset{ }^{\dot{w} g_{0}} P_{J}=\dot{w} g_{0} g P_{J} .
$$

So ${ }^{g_{1}} P$ contains some Levi of $P \cap P^{\prime}$. We have that

$$
\begin{aligned}
g_{1}^{-1}\left({ }^{g_{1}} P\right)^{(\dot{w} \dot{y}-1} P_{\left.\operatorname{Ad}\left(y_{1}\right) \delta\left(J_{1}\right)\right)} g_{1} & ={ }^{g^{-1}} P^{\left(g_{0}^{-1} \dot{y}^{-1} P_{\operatorname{Ad}(y) \delta\left(J_{1}\right)}\right)}={ }^{g^{-1}} P_{J \cap \delta^{-1}} \operatorname{Ad}\left(y^{-1}\right) \operatorname{Ad}(y) \delta\left(J_{1}\right) \\
& ={ }^{g^{-1}} P_{J_{1}}=P_{J_{1}} .
\end{aligned}
$$

Thus $\alpha(z)=z^{\prime}$. The lemma is proved.

Proposition 1.7. We have that

$$
\tilde{Z}_{J, y, \delta}^{w}=G_{\text {diag }} \cdot[\widetilde{J, w, 1}]_{y, \delta}=G_{\text {diag }} \cdot\left(P_{J},{ }^{\dot{w} \dot{y}^{-1}} P_{J^{\prime}}, U_{\dot{w} \dot{y}-1} P_{J^{\prime}} \dot{w} g_{0}\left(B \cap L_{J_{\infty}}\right) U_{P_{J}}\right) .
$$

Proof. It is easy to see that $\tilde{Z}_{J_{\infty}, w, \delta}^{w}=G_{\text {diag }}\left(\dot{w}, L_{J_{\infty}}\right) \cdot \tilde{h}_{J_{\infty}, w, \delta}$. Thus for any $b \in B$, $\alpha^{n}\left((\dot{w}, b) \cdot \tilde{h}_{J, y, \delta}\right) \in \tilde{Z}_{J_{\infty}, w, \delta}^{w}$ for sufficiently large $n$. Therefore, $G_{\text {diag }}(\dot{w}, B) \cdot \tilde{h}_{J, y, \delta} \subset$ $\tilde{Z}_{J, y, \delta}^{w}$.

Note that $\dot{w} g_{0}$ normalizes $\left(L_{J_{\infty}}\right)$ and $\left(L_{J_{\infty}}\right) \cap B$. Thus

$$
\dot{w} g_{0} L_{J_{\infty}}=\left\{l \dot{w} g_{0} b l^{-1} \mid l \in L_{J_{\infty}}, b \in L_{J_{\infty}} \cap B\right\} .
$$

Hence any element in $\tilde{Z}_{J_{\infty}, w, \delta}^{w}$ is $G$-conjugate to $(\dot{w}, l) \cdot \tilde{h}_{J_{\infty}, w, \delta}$ for some $l \in L_{J_{\infty}} \cap B$. Now let $z \in \tilde{Z}_{J, y, \delta}^{w}$. Then $\vartheta(z)$ is $G$-conjugate to $(\dot{w}, l) \cdot \tilde{h}_{J_{\infty}, w, \delta}$ for some $l \in L_{J_{\infty}} \cap B$. Set $z^{\prime}=(\dot{w}, l) \cdot \tilde{h}_{J, y, \delta} \in \tilde{Z}_{J, y, \delta}^{w}$. Then $\vartheta\left(z^{\prime}\right)$ lies in the same $G$-orbit as $\vartheta(z)$. Since $\vartheta$ induces a bijection from the set of $G$-orbits on $\tilde{Z}_{J, y, \delta}^{w}$ to the set of $G$-orbits on $\tilde{Z}_{J_{\infty}, w, \delta}^{w}, z$ is $G$-conjugate to $z^{\prime}$ and $\left.\tilde{Z}_{J, y, \delta}^{w}=G_{d i a g}\left(\dot{w}, B \cap L_{J_{\infty}}\right)\right) \cdot \tilde{h}_{J, y, \delta}$. The proposition is proved. 
1.8. In [L4, 8.20], Lusztig showed that $\tilde{Z}_{J, y, \delta}^{w}$ is an iterated affine space bundle over a fibre bundle over $\mathcal{P}_{J_{\infty}}$ with fibres isomorphic to $L_{J_{\infty}}$. In proposition 1.10, we will prove a similar (but more explicit) result, which will be used to establish the cellular decomposition. Before doing that, we include the following result (see [SL, page 26, lemma 4]) which we will use in the proof of proposition 1.10 and proposition 5.5.

Lemma 1.9. Let $H$ be a closed subgroup of $G$ and $\Phi: X \rightarrow G / H$ be a $G$-equivariant morphism from the $G$-variety $X$ to the homogeneous space $G / H$. Let $E \subset X$ be the fiber $\Phi^{-1}(H)$. Then $E$ will be stabilized by $H$ and the map $\Psi: G \times_{H} E \rightarrow X$ sending $(g, e)$ to $g \cdot e$ defines an isomorphism of $G$-varieties.

Proposition 1.10. For $a \in W$, set $U_{a}=U \cap{ }^{\dot{a}} U^{-}$. Set

$$
\tilde{L}_{J, y, d}^{w}=\left(L_{J_{\infty}}, L_{J_{\infty}}\right)(\dot{w}, 1) \cdot \tilde{h}_{J, y, \delta} .
$$

Then we have the following results:

(1) $\tilde{Z}_{J, y, \delta}^{w}$ is isomorphic to $G \times_{P_{J_{\infty}}}\left(\left(P_{J_{\infty}}\right) \cdot \tilde{L}_{J, y, \delta}^{w}\right)$.

(2) $\left(P_{J_{\infty}}\right) \cdot \tilde{L}_{J, y, \delta}^{w}=(B \times B) \cdot \tilde{L}_{J, y, \delta}^{w} \cong\left(U \cap \dot{w}_{0}^{J \infty} \dot{w} \dot{y}^{-1} \dot{w}_{0}^{J^{\prime}} U^{-}\right) \times \tilde{L}_{J, y, \delta}^{w}$, where $\tilde{L}_{J, y, \delta}^{w}$ is isomorphic to $L_{J_{\infty}}$.

(3) $G_{\text {diag }}(\dot{w} T, 1) \cdot \tilde{h}_{J, y, \delta}$ is dense in $\tilde{Z}_{J, y, \delta}^{w}$.

Proof. It is easy to see that $\tilde{L}_{J, y, \delta}^{w}=\left(\dot{w}, L_{J_{\infty}}\right) \cdot \tilde{h}_{J, y, \delta}$ is isomorphic to $L_{J_{\infty}}$. By proposition 1.7, $\tilde{Z}_{J, y, \delta}^{w}=G_{d i a g} \cdot \tilde{L}_{J, y, \delta}^{w}$. Consider the $G$-equivariant map $p$ : $\tilde{Z}_{J_{\infty}, w, \delta}^{w} \rightarrow \mathcal{P}_{J_{\infty}}$ defined by $p(P, P, \gamma)=P$ for $(P, P, \gamma) \in \tilde{Z}_{J_{\infty}, w, \delta}^{w}$. For $l \in L_{J_{\infty}}$ and $g \in G$, if $p \circ \vartheta\left((g, g)(\dot{w}, l) \cdot \tilde{h}_{J, y, \delta}\right)=P_{J_{\infty}}$, then $g \in P_{J_{\infty}}$. Thus $\left(P_{J_{\infty}}\right)_{d i a g} \cdot \tilde{L}_{J, y, \delta}^{w}=$ $(p \circ \vartheta)^{-1}\left(P_{J_{\infty}}\right)$.

Assume that $(g, g)\left(\dot{w}, l_{1}\right) \cdot \tilde{h}_{J, y, \delta}^{w}=\left(\dot{w}, l_{2}\right) \cdot \tilde{h}_{J, y, \delta}^{w}$ for $g \in G$ and $l_{1}, l_{2} \in L_{J_{\infty}}$. Then ${ }^{g} P_{J_{\infty}}=p \circ \vartheta\left((g, g)\left(\dot{w}, l_{1}\right) \cdot \tilde{h}_{J, y, \delta}^{w}\right)=p \circ \vartheta\left(\left(\dot{w}, l_{2}\right) \cdot \tilde{h}_{J, y, \delta}^{w}\right)=P_{J_{\infty}}$. So $g \in P_{J_{\infty}}$. Part (1) is proved.

We have that $(B \times B) \cdot \tilde{L}_{J, y, \delta}^{w}=(B)_{\operatorname{diag}}(1, B) \cdot \tilde{L}_{J, y, \delta}^{w}$ and $p \circ \vartheta\left((1, B) \cdot \tilde{L}_{J, y, \delta}^{w}\right)=$ $P_{J_{\infty}}$. Thus $(B \times B) \cdot \tilde{L}_{J, y, \delta}^{w} \subset(B)_{\text {diag }}\left(P_{J_{\infty}}\right)_{\text {diag }} \tilde{L}_{J, y, \delta}^{w}=\left(P_{J_{\infty}}\right)_{d i a g} \tilde{L}_{J, y, \delta}^{w}$. On the other hand, $\left(P_{J_{\infty}}\right)_{\text {diag }} \tilde{L}_{J, y, \delta}^{w} \subset\left(P_{J_{\infty}}, P_{J_{\infty}}\right) \cdot \tilde{L}_{J, y, \delta}^{w}=(B \times B) \cdot \tilde{L}_{J, y, \delta}^{w}$. Hence $\left(P_{J_{\infty}}\right)$. $\tilde{L}_{J, y, \delta}^{w}=(B \times B) \cdot \tilde{L}_{J, y, \delta}^{w}$.

Now consider $\pi:\left(U \cap \dot{w}_{0}^{J \infty} \dot{w}^{-1} \dot{w}_{0}^{J^{\prime}} U^{-}\right) \times \tilde{L}_{J, y, \delta}^{w} \rightarrow(B \times B) \cdot \tilde{L}_{J, y, \delta}^{w}$ defined by $\pi(u, l)=(u, 1) l$ for $u \in U \cap \dot{w}_{0}^{J \infty} \dot{w} \dot{y}^{-1} \dot{w}_{0}^{J^{\prime}} U^{-}$and $l \in \tilde{L}_{J, y, \delta}^{w}$.

Note that $\left(1, B L_{J_{\infty}}\right) \cdot \tilde{h}_{J, y, \delta}=\left(1, U_{P_{J}} L_{J_{\infty}} U_{J}\right) \cdot \tilde{h}_{J, y, \delta}=\left(U_{\delta(J)}, L_{J_{\infty}}\right) \cdot \tilde{h}_{J, y, \delta}$. Since $w \in W^{\delta(J)}, B \dot{w} U_{\delta(J)}=B \dot{w}=U_{P_{J_{\infty}}} L_{J_{\infty}} \dot{w}$. Hence

$$
\left(B \dot{w}, B L_{J_{\infty}}\right) \cdot \tilde{h}_{J, y, \delta}=\left(U_{P_{J_{\infty}}} \dot{w} L_{\delta\left(J_{\infty}\right)}, L_{J_{\infty}}\right) \cdot \tilde{h}_{J, y, \delta}=\left(U_{P_{J_{\infty}}} \dot{w}, L_{J_{\infty}}\right) \cdot \tilde{h}_{J, y, \delta} .
$$

Since $w y^{-1} \in W^{J^{\prime}}$ and $\operatorname{Ad}\left(y w^{-1}\right) J_{\infty} \subset J^{\prime}$, then

$$
\begin{aligned}
U_{P_{J_{\infty}}} & =\left(U_{P_{J_{\infty}}} \cap \dot{w} \dot{y}^{-1} \dot{w}_{0}^{J^{\prime}} U^{-}\right)\left(U_{P_{J_{\infty}}} \cap \dot{w} \dot{y}^{-1} U_{P_{J^{\prime}}}\right) \\
& =\left(U \cap \dot{w}_{0}^{J \infty} \dot{w} \dot{y}^{-1} \dot{w}_{0}^{J^{\prime}} U^{-}\right)\left(U_{P_{J_{\infty}}} \cap{ }^{\dot{w} \dot{y}^{-1}} U_{P_{J^{\prime}}}\right) .
\end{aligned}
$$

Therefore, $\left(B \dot{w}, B L_{J_{\infty}}\right) \cdot \tilde{h}_{J, y, \delta}=\left(\left(U \cap \dot{w}_{0}^{J_{\infty}} \dot{w} \dot{y}^{-1} \dot{w}_{0}^{J^{\prime}} U^{-}\right) \dot{w}, L_{J_{\infty}}\right) \cdot \tilde{h}_{J, y, \delta}$. So $\pi$ is surjective. 
Let $u \in U \cap \dot{w}_{0}^{J \infty} \dot{w} \dot{y}^{-1} \dot{w}_{0}^{J^{\prime}} U^{-}$and $l_{1}, l_{2} \in L_{J_{\infty}}$. Assume that $(u, 1)\left(\dot{w}, l_{1}\right) \cdot \tilde{h}_{J, y, \delta}=$ $\left(\dot{w}, l_{2}\right) \cdot \tilde{h}_{J, y, \delta}$. Note that the isotropy subgroup of $G \times G$ at the point $(\dot{w}, 1) \cdot \tilde{h}_{J, y, \delta}$ is $\left\{\left(U_{\dot{w} \dot{y}-1} P_{J^{\prime}} l^{\prime}, U_{P_{J}} g_{0}^{-1} \dot{w}^{-1} l^{\prime} \dot{w} g_{0}\right) \mid l^{\prime} \in{ }^{\dot{w}} L_{\delta(J)}\right\}$.

Thus $u \in U_{\dot{w} \dot{y}-1} P_{J^{\prime}} l^{\prime}$ and $l_{2}^{-1} l_{1} \in U_{P_{J}} g_{0}^{-1} \dot{w}^{-1} l^{\prime} \dot{w} g_{0}$ for some $l^{\prime} \in{ }^{\dot{w}} L_{\delta(J)}$. Then $l^{\prime} \in L_{J_{\infty}}$ and $u=1$. Thus $\pi$ is injective.

In fact, we can show that the bijective morphism $\pi$ is an isomorphism. The verification is omitted.

Part (3) can be proved in the same way as in [H, 2.7].

1.11. For $P \in \mathcal{P}_{J}$, let $H_{P}$ be the inverse image of the connected center of $P / U_{P}$ under $P \rightarrow P / U_{P}$. We can regard $H_{P} / U_{P}$ as a single torus $\Delta_{J}$ independent of $P$. Then $\Delta_{J}$ acts (freely) in the natural way on $\tilde{Z}_{J, y, \delta}$ and the action commutes with the action of $G$. Moreover, each piece $\tilde{Z}_{J, y, \delta}^{w}$ is $\Delta_{J}$-stable.

Define

$$
\begin{aligned}
Z_{J, y, \delta} & =\left\{\left(P, P^{\prime}, \gamma\right) \mid P \in \mathcal{P}_{J}, P^{\prime} \in \mathcal{P}_{J^{\prime}}, \gamma \in H_{P^{\prime}} \backslash A_{y}\left(P, P^{\prime}\right) / U_{P}\right\} \\
& =\left\{\left(P, P^{\prime}, \gamma\right) \mid P \in \mathcal{P}_{J}, P^{\prime} \in \mathcal{P}_{J^{\prime}}, \gamma \in U_{P^{\prime}} \backslash A_{y}\left(P, P^{\prime}\right) / H_{P}\right\}
\end{aligned}
$$

with $G \times G$ action defined by $\left(g_{1}, g_{2}\right)\left(P, P^{\prime}, \gamma\right)=\left({ }^{g_{2}} P,{ }^{g_{1}} P^{\prime}, g_{1} \gamma g_{2}^{-1}\right)$.

Then $Z_{J, y, \delta}$ and $\Delta_{J} \backslash \tilde{Z}_{J, y, \delta}$ can be identified in the natural way as varieties with $G$-action. Set $Z_{J, y, \delta}^{w}=\Delta_{J} \backslash \tilde{Z}_{J, y, \delta}^{w}$. Then

$$
Z_{J, y, \delta}=\sqcup_{w \in W^{\delta(J)}} Z_{J, y, \delta}^{w} .
$$

We call $\left(Z_{J, y, \delta}^{w}\right)_{w \in W^{\delta(J)}}$ the $G$-stable pieces of $Z_{J, y, \delta}$. Set

$$
\begin{aligned}
h_{J, y, \delta} & =\left(P_{J},{ }^{\dot{y}^{-1}} P_{J^{\prime}}, H_{\dot{y}-1} P_{J^{\prime}} g_{0} U_{P_{J}}\right) \in Z_{J, y, \delta}, \\
L_{J, y, \delta}^{w} & =\left(L_{J_{\infty}}, L_{J_{\infty}}\right)(\dot{w}, 1) \cdot h_{J, y, \delta} .
\end{aligned}
$$

For $w \in W^{\delta(J)}$ and $v \in W$, set $[J, w, v]_{y, \delta}=(B \times B)(\dot{w}, \dot{v}) \cdot h_{J, y, \delta}$. Then as a consequence of propositions 1.7 and 1.10, we have the following result.

Proposition 1.12. For $w \in W^{\delta(J)}$, we have that

(1) $Z_{J, y, \delta}^{w}=G_{\text {diag }} \cdot[J, w, 1]_{y, \delta}$.

(2) $Z_{J, y, \delta}^{w}$ is isomorphic to $G \times_{P_{J_{\infty}}}\left(\left(P_{J_{\infty}}\right) \cdot L_{J, y, \delta}^{w}\right)$.

(3) $\left(P_{J_{\infty}}\right) \cdot L_{J, y, \delta}^{w}=(B \times B) \cdot L_{J, y, \delta}^{w} \cong\left(U \cap \dot{w}_{0}^{J \infty} \dot{w} \dot{y}^{-1} \dot{w}_{0}^{J^{\prime}} U^{-}\right) \times L_{J, y, \delta}^{w}$, where $L_{J, y, \delta}^{w}$ is isomorphic to $L_{J_{\infty}} / Z\left(L_{J}\right)$.

(4) $G_{\text {diag }}(\dot{w} T, 1) \cdot h_{J, y, \delta}$ is dense in $Z_{J, y, \delta}^{w}$.

\section{Compactification through the Fibres}

2.1. For any connected, semi-simple algebraic group of adjoint type, De Concini and Procesi introduced its wonderful compactification $\bar{G}$ (see [DP). It is an irreducible, projective smooth $G \times G$-variety. The $G \times G$-orbits $Z_{J}$ of $\bar{G}$ are indexed by the subsets $J$ of $I$. Moreover, $Z_{J}=(G \times G) \times_{P_{J}^{-} \times P_{J}} G_{J}$, where $P_{J}^{-} \times P_{J}$ acts on the right on $G \times G$ and on the left on $G_{J}$ by $(q, p) \cdot z=\pi_{P_{J}^{-}}(q) z \pi_{P_{J}}(p)$. Denote by $h_{J}$ the image of $(1,1,1)$ in $Z_{J}$. We will identify $Z_{J}$ with $Z_{J, w_{0} w_{0}^{J}, i d}$ and $h_{J}$ with $h_{J, w_{0} w_{0}^{J}, i d}$, where $i d$ is the identity map on $I$ (see [H, 2.5]). 
Let us consider the $B \times B$-orbits on $\bar{G}$. For any $J \subset I, u \in W^{J}$ and $v \in W$, set $[J, u, v]=(B \times B)(\dot{u}, \dot{v}) \cdot h_{J}$. Then $\bar{G}=\bigsqcup_{J \subset I} \bigsqcup_{x \in W^{J}, w \in W}[J, x, w]$. The following result is due to Springer (see [S, 2.4]).

Theorem. Let $x \in W^{J}, x^{\prime} \in W^{K}, w, w^{\prime} \in W$. Then $\left[K, x^{\prime}, w^{\prime}\right]$ is contained in the closure of $[J, x, w]$ if and only if $K \subset J$ and there exists $u \in W_{K}, v \in W_{J} \cap W^{K}$ with $x v u^{-1} \leqslant x^{\prime}, w^{\prime} u \leqslant w v$ and $l(w v)=l(w)+l(v)$. In particular, for $J \subset I$ and

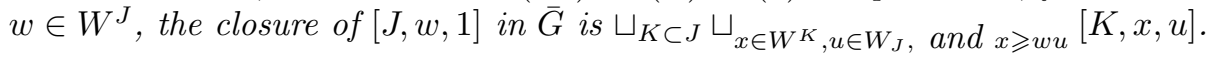

2.2. We have defined $Z_{J, y, \delta}$ in section 1.11. As we have seen, $Z_{J, y, \delta}$ is a locally trivial fibre bundle over $\mathcal{P}_{J} \times \mathcal{P}_{J^{\prime}}$ with fibres isomorphic to $L_{J} / Z\left(L_{J}\right)$. Note that $L_{J} / Z\left(L_{J}\right)$ is a connected, semi-simple algebraic group of adjoint type. Thus we can define the wonderful compactification $\overline{L_{J} / Z\left(L_{J}\right)}$ of $L_{J} / Z\left(L_{J}\right)$. In this section, we will define $Z_{J, y, \delta}$, which is a locally trivial fibre bundle over $\mathcal{P}_{J} \times \mathcal{P}_{J^{\prime}}$ with fibres isomorphic to $\overline{\overline{L_{J} / Z\left(L_{J}\right)}}$.

2.3. We keep the notation of section 1.3. Fix $g \in A_{y}\left(P, P^{\prime}\right)$. Then $A_{y}\left(P, P^{\prime}\right) g^{-1}=$ $P^{\prime} U_{g}$ (see [L4, 8.9]). Set

$$
L_{P, P^{\prime}, g}={ }^{g} P \cap P^{\prime} / H_{g} P \cap P^{\prime} .
$$

Let $\Phi_{g}: H_{P^{\prime}} \backslash A_{y}\left(P, P^{\prime}\right) / H_{P} \rightarrow L_{P, P^{\prime}, g}$ be the morphism defined by

$$
H_{P^{\prime}} \backslash A_{y}\left(P, P^{\prime}\right) / H_{P} \stackrel{\cdot g^{-1}}{\rightarrow} H_{P^{\prime}} \backslash A_{y}\left(P, P^{\prime}\right) g^{-1} / H_{g} P \stackrel{i}{\leftarrow} L_{P, P^{\prime}, g},
$$

where $i$ is the obvious isomorphism.

The $P \times P^{\prime}$ action on $H_{P^{\prime}} \backslash A_{y}\left(P, P^{\prime}\right) / H_{P}$ induces a $P \times P^{\prime}$ action on $L_{P, P^{\prime}, g}$. Now for $g, g^{\prime} \in A_{y}\left(P, P^{\prime}\right)$, set $\Phi_{g, g^{\prime}}=\Phi_{g^{\prime}} \Phi_{g}^{-1}: L_{P, P^{\prime}, g} \stackrel{\simeq}{\rightarrow} L_{P, P^{\prime}, g^{\prime}}$. Then $\Phi_{g, g^{\prime}}$ is compatible with the $P \times P^{\prime}$ action. Moreover, $\left(L_{P, P^{\prime}, g}, \Phi_{g, g^{\prime}}\right)$ forms an inverse system and

$$
H_{P^{\prime}} \backslash A_{y}\left(P, P^{\prime}\right) / H_{P}=\lim _{\leftarrow} L_{P, P^{\prime}, g} .
$$

Note that $L_{P, P^{\prime}, g}$ is a semi-simple group of adjoint type. Then we can define the De Concini-Procesi compactification $\overline{L_{P, P^{\prime}, g}}$ of $L_{P, P^{\prime}, g}$. The $P \times P^{\prime}$ action on $L_{P, P^{\prime}, g}$ can be extended in the unique way to a $P \times P^{\prime}$ action on $\overline{L_{P, P^{\prime}, g}}$. The isomorphism $\Phi_{g, g^{\prime}}: L_{P, P^{\prime}, g} \stackrel{\simeq}{\rightarrow} L_{P, P^{\prime}, g^{\prime}}$ can be extended in the unique way to an isomorphism from $\overline{L_{P, P^{\prime}, g}}$ onto $\overline{L_{P, P^{\prime}, g^{\prime}}}$. We will also denote this isomorphism by $\Phi_{g, g^{\prime}}$. It is easy to see that this isomorphism is compatible with the $P \times P^{\prime}$ action. Now $\left.\overline{\left(L_{P, P^{\prime}, g}\right.}, \Phi_{g, g^{\prime}}\right)$ forms an inverse system. Define

$$
\overline{H_{P^{\prime}} \backslash A_{y}\left(P, P^{\prime}\right) / H_{P}}=\lim _{\leftarrow} \overline{L_{P, P^{\prime}, g}} .
$$

We also obtain a $P \times P^{\prime}$ action on $\overline{H_{P^{\prime}} \backslash A_{y}\left(P, P^{\prime}\right) / H_{P}}$. Thus we can identify $\overline{H_{P^{\prime}} \backslash A_{y}\left(P, P^{\prime}\right) / H_{P}}$ with $\overline{L_{P, P^{\prime}, g}} g$ as varieties with $P \times P^{\prime}$ action.

Remark. $\overline{H_{P^{\prime}} \backslash A_{y}\left(P, P^{\prime}\right) / H_{P}}$ is isomorphic to $\overline{L_{P, P^{\prime}, g}}$ as a variety. However, we are also concerned with the $P^{\prime} \times P$ action. In this case, $\overline{H_{P^{\prime}} \backslash A_{y}\left(P, P^{\prime}\right) / H_{P}}$ is regarded as $\overline{L_{P, P^{\prime}, g}} g$ with a "twisted" $P^{\prime} \times P$ action. 
2.4. In this section, we will consider a special case, namely, $P=P^{\prime}=G^{0}$. In this case, $A_{y}\left(P, P^{\prime}\right)=G^{1}$ and we will identify $H_{G} \backslash A_{y}(G, G) / H_{G}$ with $G^{1}$.

Let $\mathcal{V}_{G}$ be the projective variety whose points are the $\operatorname{dim}(G)$-dimensional Lie subalgebras of $\operatorname{Lie}(G \times G)$. The $\hat{G} \times \hat{G}$ action on $\operatorname{Lie}(G \times G)$ which is defined by $\left(g_{1}, g_{2}\right) \cdot(a, b)=\left(\operatorname{Ad}\left(g_{2}\right) a, \operatorname{Ad}\left(g_{1}\right) b\right)$ for $g_{1}, g_{2} \in \hat{G}$ and $a, b \in \operatorname{Lie}(G)$ induces a $\hat{G} \times \hat{G}$ action on $\mathcal{V}_{G}$. To each $g \in \hat{G}$, we associate a $\operatorname{dim}(G)$-dimensional subspace $V_{g}=\{(a, \operatorname{Ad}(g) a) \mid a \in \operatorname{Lie}(G)\}$ of $\operatorname{Lie}(G \times G)$. Then $V_{g_{1} g g_{2}^{-1}}=\left(g_{1}, g_{2}\right) \cdot V_{g}$ for $g_{1}, g, g_{2} \in \hat{G}$, and $g \mapsto V_{g}$ is an embedding $G^{1} \subset \mathcal{V}_{G}$. We denote the image by $i\left(G^{1}\right)$.

If $G^{1}=G$, then the closure of $i(G)$ in $\mathcal{V}_{G}$ is $\bar{G}$ (see [DP). Note that $V_{g g_{0}}=$ $\left(1, g_{0}^{-1}\right) V_{g}$ for all $g \in G$. Thus $i\left(G^{1}\right)=\left(1, g_{0}^{-1}\right) i(G)$. Hence the closure of $i\left(G^{1}\right)$ in $\mathcal{V}_{G}$ is $\left(1, g_{0}^{-1}\right) \bar{G}$, which is just $\bar{G}^{1}$ defined above.

Remark. In [L4, 12.3], Lusztig defined the compactification of $G^{1}$ to be the closure of $i\left(G^{1}\right)$ in $\mathcal{V}_{G}$. As we have seen, our definition coincides with his definition.

2.5. In [L4, 12.3], Lusztig showed that

$$
\bar{G}^{1}=\sqcup_{J \subset I} Z_{J, w_{0} w_{0}^{\delta(J)}, \delta}=\sqcup_{J \subset I} \sqcup_{w \in W^{\delta(J)}} Z_{J, w_{0} w_{0}^{\delta(J)}, \delta}^{w},
$$

where the base point $h_{J, w_{0} w_{0}^{\delta(J)}, \delta}=\left(P_{J}, P_{\delta(J)}^{-}, H_{P_{\delta(J)}^{-}} g_{0} H_{P_{J}}\right)$ is identified with the $\operatorname{dim}(G)$-dimensional subalgebra $\left\{\left(l u, g_{0} l g_{0}^{-1} u^{\prime}\right) \mid l \in L_{J}, u \in U_{P_{J}}, u^{\prime} \in U_{P_{\delta(J)}^{-}}\right\}$of $\operatorname{Lie}(G \times G)$. We will simply write $h_{J, w_{0} w_{0}^{\delta(J)}, \delta}$ as $h_{J, \delta},[J, w, v]_{w_{0} w_{0}^{\delta(J)}, \delta}$ as $[J, w, v]_{\delta}$ and $Z_{J, w_{0} w_{0}^{\delta(J)}, \delta}^{w}$ as $Z_{J, \delta}^{w}$. We call $\left(Z_{J, \delta}^{w}\right)_{J \subset I, w \in W^{\delta(J)}}$ the $G$-stable pieces of $\bar{G}^{1}$. If $G^{1}=G$, then $h_{J, i d}=h_{J}$ and $[J, w, v]_{i d}=[J, w, v]$.

Note that $h_{J}$ corresponds to the $\operatorname{dim}(G)$-dimensional subalgebra $\left\{\left(l u, l u^{\prime}\right) \mid l \in\right.$ $\left.L_{J}, u \in U_{P_{J}}, u^{\prime} \in U_{P_{J}^{-}}\right\}$of $\operatorname{Lie}(G \times G)$. Thus $h_{J, \delta}=\left(1, g_{0}^{-1}\right) h_{\delta(J)}$. Hence

$$
\begin{aligned}
& {[J, w, v]_{\delta}=(B \times B)(\dot{w}, \dot{v}) \cdot h_{J, \delta}=(B \times B)(\dot{w}, \dot{v})\left(1, g_{0}^{-1}\right) \cdot h_{J}} \\
& =\left(1, g_{0}^{-1}\right)(B \times B)(\dot{w}, \delta(v)) \cdot h_{\delta(J)}=\left(1, g_{0}^{-1}\right)[\delta(J), w, \delta(v)] .
\end{aligned}
$$

Thus we have the following result.

Proposition. Let $J \subset I$ and $w \in W^{\delta(J)}$. Then the closure of $[J, w, 1]_{\delta}$ in $\bar{G}^{1}$ is $\sqcup_{K \subset J} \sqcup_{x \in W^{\delta(K)}, u \in W_{J}}$, and $x \geqslant w \delta(u)[K, x, u]_{\delta}$.

2.6. Define

$$
\underline{Z_{J, y, \delta}}=\left\{\left(P, P^{\prime}, \gamma\right) \mid P \in \mathcal{P}_{J}, P^{\prime} \in \mathcal{P}_{J^{\prime}}, \gamma \in \overline{H_{P^{\prime}} \backslash A_{y}\left(P, P^{\prime}\right) / H_{P}}\right\}
$$

with $G \times G$ action defined by $\left(g_{1}, g_{2}\right)\left(P, P^{\prime}, \gamma\right)=\left({ }^{g_{2}} P,{ }^{g_{1}} P^{\prime}, g_{1} \gamma g_{2}^{-1}\right)$.

Set $P=P_{J}$ and $P^{\prime}=\dot{y}^{-1} P_{J^{\prime}}$. Then $\overline{A_{y}\left(P, P^{\prime}\right)}$ can be identified with $\overline{L_{P, P^{\prime}, g_{0}}} g_{0}$ as varieties with $P^{\prime} \times P$ action. Moreover, we have a canonical isomorphism between $\overline{L_{P, P^{\prime}, g_{0}}}$ and $\overline{L_{\delta(J)}}$. For $K \subset J$, I will identify $h_{\delta(K)} g_{0}$ with the corresponding element in $\overline{A_{y}\left(P, P^{\prime}\right)}$.

Then the $G \times G$-orbits in $Z_{J, y, \delta}$ are in one-to-one correspondence with the subsets of $J$, i.e.,

$$
\underline{Z_{J, y, \delta}}=\sqcup_{K \subset J}(G \times G) \cdot\left(P, P^{\prime}, h_{\delta(K)} g_{0}\right) .
$$


Set $y_{K}=y w_{0}^{\delta(J)} w_{0}^{\delta(K)}$. Note that $U_{P_{J}}\left(L_{J} \cap U_{P_{K}}\right)=U_{P_{K}}$ and

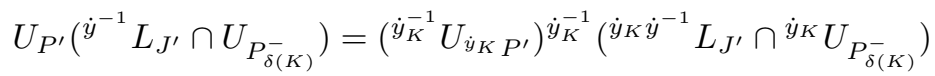

$$
\begin{aligned}
& =\dot{y}_{K}^{-1}\left(U_{P_{J^{\prime}}}\left(L_{J^{\prime}} \cap U_{P_{\mathrm{Ad}\left(y_{K}\right) \delta(K)}}\right)\right)=\dot{y}_{K}^{-1} U_{P_{\mathrm{Ad}\left(y_{K}\right) \delta(K)}} .
\end{aligned}
$$

The isotropic subgroup of $G \times G$ at $\left(P, P^{\prime}, h_{\delta(K)} g_{0}\right)$ is $\left\{\left(l_{1} u_{1}, g_{0}^{-1} l_{2} g_{0} u_{2}\right) \mid l_{1}, l_{2} \in\right.$ $\left.L_{\delta(K)}, l_{1} l_{2}^{-1} \in Z\left(L_{\delta(K)}\right), u_{1} \in U_{\dot{y}_{K}^{-1} P_{\mathrm{Ad}_{\left(y_{K}\right) \delta(K)}}}, u_{2} \in U_{P_{K}}\right\}$. Now set $Q=P_{K}, Q^{\prime}=$ $\dot{y}_{K}^{-1} P_{\operatorname{Ad}\left(y_{K}\right) \delta(K)}$ and $\gamma=H_{Q^{\prime}} g_{0} H_{Q}$. Then $\operatorname{pos}\left(Q^{\prime},{ }^{g_{0}} Q\right)=y_{K}$ and $\left(Q, Q^{\prime}, \gamma\right) \in$ $Z_{K, y_{K}, \delta}$. The isotropic subgroup of $G \times G$ at $\left(P, P^{\prime}, h_{\delta(K)} g_{0}\right)$ is the same as the isotropic subgroup of $G \times G$ at $\left(Q, Q^{\prime}, \gamma\right) \in Z_{K, y_{K}, \delta}$. Thus we can identify $\left(P, P^{\prime}, h_{\delta(K)} g_{0}\right)$ with $\left(Q, Q^{\prime}, \gamma\right)$ and $(G \times G) \cdot\left(P, P^{\prime}, h_{\delta(K)} g_{0}\right)$ with $Z_{K, y_{K}, \delta}$ as varieties with $G \times G$ action. In other words,

$$
\underline{Z_{J, y, \delta}}=\sqcup_{K \subset J} Z_{K, y w_{0}^{\delta(J)}} w_{0}^{\delta(K)}{ }_{, \delta} \cdot
$$

\section{PARTial order on $\mathcal{I}_{\delta}$}

In this section, we will only consider subvarieties of $G$ and for any subvariety $X$ of $G$, we denote by $\bar{X}$ the closure of $X$ in $G$.

3.1. Let $y, w \in W$. Then $y \leqslant w$ if and only if for any reduced expression $w=$ $s_{1} s_{2} \cdots s_{q}$, there exists a subsequence $i_{1}<i_{2}<\cdots<i_{r}$ of $1,2, \ldots, q$ such that $y=s_{i_{1}} s_{i_{2}} \cdots s_{i_{r}}$ (see [L2, 2.4]).

The following assertions follow from the above property:

(1) If $l(w u)=l(w)+l(u)$, then for any $w_{1} \leqslant w$ and $u_{1} \leqslant u, w_{1} u_{1} \leqslant w u$.

(2) Let $u, v \in W$ and $i \in I$. Assume that $s_{i} v<v$; then $u \leqslant v \Leftrightarrow s u \leqslant v$.

(3) Let $u, v \in W$ and $i \in I$. Assume that $u<s_{i} u$; then $u \leqslant v \Leftrightarrow u \leqslant s_{i} v$.

The assertion (1) follows directly from the above property. The proofs of assertions (2) and (3) can be found in [L2, 2.5].

3.2. It is known that $G=\sqcup_{w \in W} B \dot{w} B$ and for $w, w^{\prime} \in W, B \dot{w} B \subset \overline{B \dot{w}^{\prime} B}$ if and only if $w \leqslant w^{\prime}$. Moreover,

$$
\overline{B \dot{s}_{i} B \dot{w} B}= \begin{cases}\overline{B \dot{w} B}, & \text { if } s_{i} w<w \\ \overline{B \dot{s}_{i} \dot{w} B}, & \text { if } s_{i} w>w .\end{cases}
$$

Similarly, $G=\sqcup_{w \in W} B \dot{w} B^{-}$and for $w, w^{\prime} \in W, B \dot{w} B^{-} \subset \overline{B \dot{w}^{\prime} B^{-}}$if and only if $w \geqslant w^{\prime}$. Moreover,

$$
\overline{B \dot{s}_{i} B \dot{w} B^{-}}= \begin{cases}\overline{B \dot{w} B^{-}}, & \text {if } s_{i} w>w ; \\ \overline{B \dot{s}_{i} \dot{w} B^{-}}, & \text {if } s_{i} w<w .\end{cases}
$$

Lemma 3.3. Let $u, w \in W$. Then

(1) The subset $\{v w \mid v \leqslant u\}$ of $W$ contains a unique minimal element $y$. Moreover, $l(y)=l(w)-l\left(y w^{-1}\right)$ and $\overline{B \dot{u} B \dot{w} B^{-}}=\overline{B \dot{y} B^{-}}$.

(2) The subset $\{v w \mid v \leqslant u\}$ of $W$ contains a unique maximal element $y^{\prime}$. Moreover, $l\left(y^{\prime}\right)=l(w)+l\left(y^{\prime} w^{-1}\right)$ and $\overline{B \dot{u} B \dot{w} B}=\overline{B \dot{y}^{\prime} B}$.

Proof. We will only prove part (1). Part (2) can be proved in the same way.

For any $v \leqslant u, B \dot{v} \subset \overline{B \dot{u} B}$. Thus $\overline{B \dot{v} \dot{w} B^{-}} \subset \overline{B \dot{u} B} \dot{w} B^{-} \subset \overline{B \dot{u} B \dot{w} B^{-}}$. On the other hand, $\overline{B \dot{u} B \dot{w} B^{-}}$is an irreducible, closed, $B \times B^{-}$-stable subvariety of $G$. Thus there exists $y \in W$, such that $\overline{B \dot{u} B \dot{w} B^{-}}=\overline{B \dot{y} B^{-}}$. Since $B \dot{v} \dot{w} B^{-} \subset \overline{B \dot{y} B^{-}}$, 
we have that $v w \geqslant y$. Now it suffices to prove that $y=v w$ for some $v \leqslant u$ with $l(v w)=l(w)-l(v)$.

We argue by induction on $l(u)$. If $l(u)=0$, then $u=1$ and the statement is clear. Assume now that $l(u)>0$. Then there exists $i \in I$, such that $s_{i} u<u$. We denote $s_{i} u$ by $u^{\prime}$. Now

$$
\overline{B \dot{u} B \dot{w} B^{-}}=\overline{B \dot{s}_{i} B \dot{u}^{\prime} B \dot{w} B^{-}}=\overline{B \dot{s}_{i} \overline{B \dot{u}^{\prime} B \dot{w} B^{-}}} .
$$

By induction hypothesis, there exists $v^{\prime} \leqslant u^{\prime}$, such that $l\left(v^{\prime} w\right)=l(w)-l\left(v^{\prime}\right)$ and $\overline{B \dot{u}^{\prime} B \dot{w} B^{-}}=\overline{B \dot{v}^{\prime} \dot{w} B^{-}}$. Thus

$$
\overline{B \dot{s}_{i} \overline{B \dot{u}^{\prime} B \dot{w} B^{-}}}=\overline{B \dot{s}_{i} \overline{B \dot{v}^{\prime} \dot{w} B^{-}}}=\overline{B \dot{s}_{i} B \dot{v}^{\prime} \dot{w} B^{-}}= \begin{cases}\overline{B \dot{v}^{\prime} \dot{w} B^{-}}, & \text {if } s_{i} v^{\prime} w>v^{\prime} w \\ \overline{B \dot{s}_{i} \dot{v}^{\prime} \dot{w} B^{-}}, & \text {if } s_{i} v^{\prime} w<v^{\prime} w\end{cases}
$$

Note that $s_{i} u<u$ and $v^{\prime} \leqslant s_{i} u<u$. Thus $s_{i} v^{\prime} \leqslant u$. Moreover, if $s_{i} v^{\prime} w<v^{\prime} w$, then $l\left(s_{i} v^{\prime} w\right)=l\left(v^{\prime} w\right)-1=l(w)-l\left(v^{\prime}\right)-1$. Thus we have that $l\left(s_{i} v^{\prime}\right)=l(v)+1$ and $l\left(s_{i} v^{\prime} w\right)=l(w)-l\left(s_{i} v^{\prime}\right)$. Therefore, the statement holds for $u$.

Corollary 3.4. Let $u, w, w^{\prime} \in W$ with $w^{\prime} \leqslant w$. Then

(1) There exists $v \leqslant u$, such that $v w^{\prime} \leqslant u w$.

(2) There exists $v^{\prime} \leqslant u$, such that $u w^{\prime} \leqslant v^{\prime} w$.

Proof. Let $v \leqslant u$ be the element of $W$ such that $v w^{\prime}$ is the unique minimal element in $\left\{v^{\prime} w^{\prime} \mid v^{\prime} \leqslant u\right\}$. Then $\overline{B \dot{u} B \dot{w}^{\prime} B^{-}}=\overline{B \dot{v} \dot{w}^{\prime} B^{-}}$. Since $w^{\prime} \leqslant w$, we have that $B \dot{w} B^{-} \subset \overline{B \dot{w}^{\prime} B^{-}}$. Thus

$$
B \dot{u} \dot{w} B^{-} \subset B \dot{u} B \dot{w} B^{-} \subset B \dot{u} \overline{B \dot{w}^{\prime} B^{-}} \subset \overline{B \dot{u} B \dot{w}^{\prime} B^{-}}=\overline{B \dot{v} \dot{w}^{\prime} B^{-}} .
$$

So $u w \geqslant v w^{\prime}$. Thus part (1) is proved. Part (2) can be proved in the same way.

3.5. We will recall some known results about $W^{J}$.

(1) If $w \in W^{J}$ and $i \in I$, then there are three possibilities:

(a) $s_{i} w>w$ and $s_{i} w \in W^{J}$;

(b) $s_{i} w>w$ and $s_{i} w=w s_{j}$ for some $j \in J$;

(c) $s_{i} w<w$ in which case $s_{i} w \in W^{J}$.

(2) If $w \in W^{J}, v \in W_{J}$ and $K \subset J$; then $v \in W^{K}$ if and only if $w v \in W^{K}$.

(3) If $w \in{ }^{J^{\prime}} W^{J}$ and $u \in W_{J^{\prime}}$, then $u w \in W^{J}$ if and only if $u \in W^{K}$, where $K=J^{\prime} \cap \operatorname{Ad}(w) J$.

Lemma 3.6. Let $w \in{ }^{J^{\prime}} W^{J}, u \in W_{J^{\prime}}$ and $K=J^{\prime} \cap \operatorname{Ad}(w) J$; then $u w=v w u^{\prime}$ for some $v \in W_{J^{\prime}} \cap W^{K}$ and $u^{\prime} \in W_{\operatorname{Ad}\left(w^{-1}\right) K}$.

Proof. We argue by induction on $l(u)$. If $u=1$, then the statement is clear. Now assume that $u=s_{i} u_{1}$ for some $i \in J^{\prime}$ and $l\left(u_{1}\right)<l(u)$. Then by induction hypothesis, $u_{1} w=v_{1} w u_{1}^{\prime}$ for some $v_{1} \in W_{J^{\prime}} \cap W^{K}$ and $u_{1}^{\prime} \in W_{\operatorname{Ad}\left(w^{-1}\right) K}$.

If $s_{i} v_{1} w \in W^{J}$, then the statement holds for $u$. Now assume that $s_{i} v_{1} w \notin W^{J}$. Then $s_{i} v_{1} w>v_{1} w$. Hence $s_{i} v_{1}>v_{1}$. Moreover, $s_{i} v_{1} \notin W^{K}$. Thus $s_{i} v_{1}=v_{1} s_{k}$ for some $k \in K$. Note that $s_{k} w=w s_{l}$ for some $l \in \operatorname{Ad}\left(w^{-1}\right) K$. Thus the statement holds for $u$. The lemma is proved. 
3.7. Let $J \subset I$ and $w, w^{\prime} \in W$ with $l(w)=l\left(w^{\prime}\right)$. We say that $w^{\prime}$ can be obtained from $w$ via a $(J, \delta)$-cyclic shift if $w=s_{i_{1}} s_{i_{2}} \cdots s_{i_{n}}$ is a reduced expression and either (1) $i_{1} \in J$ and $w^{\prime}=s_{i_{1}} w s_{\delta\left(i_{1}\right)}$ or $(2) i_{n} \in \delta(J)$ and $w^{\prime}=s_{\delta^{-1}\left(i_{n}\right)} w s_{i_{n}}$. We say that $w$ and $w^{\prime}$ are equivalent in $J$ if there exists a finite sequence of elements $w=w_{0}, w_{1}, \ldots, w_{m}=w^{\prime}$ such that $w_{k+1}$ can be obtained from $w_{k}$ via a $(J, \delta)$-cyclic shift. (We then write $w \sim_{J, \delta} w^{\prime}$.)

Proposition 3.8. Let $(J, w) \in \mathcal{I}_{\delta}$ and $w^{\prime} \in W$. The following conditions on $w^{\prime}$ are equivalent:

(1) $w^{\prime} \geqslant u^{-1} w \delta(u)$ for some $u \in W_{J}$.

(2) $w^{\prime} \geqslant u^{-1} w \delta(v)$ for some $u \leqslant v \in W_{J}$.

(3) $w^{\prime} \geqslant w_{1}$ for some $w_{1} \sim_{J, \delta} w$.

Proof. The implication $(1) \Rightarrow(2)$ is trivial. The implication $(3) \Rightarrow(1)$ follows from the definition. We now prove the implication $(2) \Rightarrow(3)$ by induction on $|J|$. Assume that the implication holds for all $J^{\prime} \subset I$ with $\left|J^{\prime}\right|<|J|$. Then we prove that the implication holds for $J$ by induction on $l(v)$.

Set $w=x y$ with $x \in W_{J}$ and $y \in{ }^{J} W^{\delta(J)}$. Set $K=J \cap \delta^{-1} \operatorname{Ad}\left(y^{-1}\right) J, v=v_{1} v_{2}$ with $v_{1} \in W_{K}, v_{2} \in{ }^{K} W$ and $u=u_{1} u_{2}$ with $u_{1} \leqslant v_{1}, u_{2} \leqslant v_{2}$ and $l(u)=$ $l\left(u_{1}\right)+l\left(u_{2}\right)$. There are two cases.

Case 1. $u_{2}=v_{2}=1$.

In this case, $u, v \in W_{K}$ and $w \in W^{\delta(K)}$. If $|K|<|J|$, then by induction hypothesis, $u^{-1} w \delta(v) \geqslant w_{1}$ for some $w_{1} \sim_{K, \delta} w$. If $K=J$, then since $w=x y \in$ $W^{\delta(J)}$, we have that $x=1$. Thus $u^{-1} w \delta(v) \geqslant w$. The implication is proved in this case.

Case 2. $v_{2} \neq 1$.

In this case, $l\left(v_{1}\right)<l(v)$. By induction hypothesis, there exists $w_{1} \sim_{J, \delta} w$, such that $w_{1} \leqslant u_{1}^{-1} w \delta\left(v_{1}\right)$. Let $u_{3} \leqslant u_{2}$ be the element in $W$ such that $u_{3}^{-1} w_{1}$ is the unique minimal element in $\left\{\left(u^{\prime}\right)^{-1} w_{1} \mid u^{\prime} \leqslant u_{2}\right\}$. Then $l\left(u_{3}^{-1} w_{1}\right)=l\left(w_{1}\right)-l\left(u_{3}\right)$ and $u_{3}^{-1} w_{1} \leqslant u_{2}^{-1} u_{1}^{-1} w \delta\left(v_{1}\right)=u^{-1} w \delta\left(v_{1}\right)$. By lemma 3.6, $u^{-1} w=a b$ for some $a \in$ $W^{\delta(J)}$ and $b \in W_{\delta(K)}$. Thus $l\left(u^{-1} w \delta\left(v_{1} v_{2}\right)\right)=l\left(a b \delta\left(v_{1} v_{2}\right)\right)=l(a)+l\left(b \delta\left(v_{1} v_{2}\right)\right)=$ $l(a)+l\left(b \delta\left(v_{1}\right)\right)+l\left(\delta\left(v_{2}\right)\right)=l\left(a b \delta\left(v_{1}\right)\right)+l\left(\delta\left(v_{2}\right)\right)=l\left(u^{-1} w \delta\left(v_{1}\right)\right)+l\left(\delta\left(v_{2}\right)\right)$. By section $3.1, u_{3}^{-1} w_{1} \delta\left(u_{3}\right) \leqslant u^{-1} w \delta(v)$.

Now assume that $u_{3}=s_{i_{1}} s_{i_{2}} \cdots s_{i_{k}}$ and $u_{3}^{-1} w_{1}=s_{j_{1}} s_{j_{2}} \cdots s_{j_{l}}$ are reduced expressions. For $m=1,2, \ldots, k+1$, set

$$
x_{m}=\left(s_{i_{m}} s_{i_{m+1}} \cdots s_{i_{k}}\right)\left(s_{j_{1}} s_{j_{2}} \cdots s_{j_{l}}\right)\left(s_{\delta\left(i_{1}\right)} s_{\delta\left(i_{2}\right)} \cdots s_{\delta\left(i_{m-1}\right)}\right) .
$$

Then $l\left(x_{m}\right) \leqslant k+l=l\left(w_{1}\right)$ for all $m$. On the other hand, for any $m$, there exists $y_{m} \in W_{J}$, such that $x_{m}=y_{m}^{-1} w \delta\left(y_{m}\right)$. Note that $w \in W^{J}$; we have that $l\left(y_{m}^{-1} w \delta\left(y_{m}\right)\right) \geqslant l\left(w \delta\left(y_{m}\right)\right)-l\left(y_{m}^{-1}\right)=l(w)=l\left(w_{1}\right)$ for all $y_{m} \in W_{J}$. Thus $l\left(x_{m}\right)=$ $l\left(w_{1}\right)$ and $x_{m} \sim_{J, \delta} w_{1}$ for all $m$. In particular, $u_{3}^{-1} w_{1} \delta\left(u_{3}\right)=x_{k+1} \sim_{J, \delta} w_{1}$. The implication is proved in this case.

Remark. We see from the proof that $u^{-1} w \delta(v) \geqslant x^{-1} w \delta(x)$ for some $x \leqslant u$. This result will be used in the proof of lemma 5.2.

3.9. Let $(J, w) \in \mathcal{I}_{\delta}$ and $w^{\prime} \in W$; we say that $w^{\prime} \geqslant_{J, \delta} w$ if $w^{\prime}$ satisfies the equivalent conditions of proposition 3.8 (1)-(3). It is easy to see that $x \geqslant w \Rightarrow x \geqslant_{J, \delta} w \Rightarrow$ $l(x) \geqslant l(w)$. 
Now for $\left(J_{1}, w_{1}\right),\left(J_{2}, w_{2}\right) \in \mathcal{I}_{\delta}$, we say that $\left(J_{1}, w_{1}\right) \leqslant \delta\left(J_{2}, w_{2}\right)$ if $J_{1} \subset J_{2}$ and $w_{1} \geqslant_{J, \delta} w_{2}$. In the end of this section, we will show that $\leqslant$ is a partial order on $\mathcal{I}_{\delta}$. (The definition of partial order can be found in section 3.12).

Lemma 3.10. Let $J \subset I, w \in W^{J}, u \in W$ with $l(u w)=l(u)+l(w)$. Assume that $u w=x v$ with $x \in W^{J}$ and $v \in W_{J}$. Then for any $v^{\prime} \leqslant v$, there exists $u^{\prime} \leqslant u$, such that $u^{\prime} w=x v^{\prime}$.

Proof. We argue by induction on $l(u)$. If $l(u)=0$, then $u=1$ and the statement is clear. Assume now that $l(u)>0$. Then there exists $i \in I$, such that $s_{i} u<u$. We denote $s_{i} u$ by $u_{1}$. Let $u_{1} w=x_{1} v_{1}$ with $x_{1} \in W^{J}$ and $v_{1} \in W_{J}$. Then $s_{i} x_{1}>x_{1}$.

If $s_{i} x_{1} \in W^{J}$, then the lemma holds by induction hypothesis. If $s_{i} x_{1} \notin W^{J}$, then there exists $j \in J$, such that $s_{i} x_{1}=x_{1} s_{j}$. In this case, $s_{j} v_{1}>v_{1}$. Let $v^{\prime} \leqslant s_{j} v_{1}$. If $v^{\prime} \leqslant v_{1}$, then the lemma holds by induction hypothesis. If $v^{\prime} \nless v_{1}$, then $v^{\prime}=s_{j} v_{1}^{\prime}$ for some $v_{1}^{\prime} \leqslant v_{1}$. By induction hypothesis, there exists $u_{1}^{\prime} \leqslant u_{1}$, such that $u_{1}^{\prime} w=x_{1} v_{1}^{\prime}$. Thus $s_{i} u_{1}^{\prime} w=x_{1} s_{j} v_{1}^{\prime}$. The lemma holds in this case.

Lemma 3.11. Fix $J \subset I$ and $w \in W^{\delta(J)}$. For any $K \subset J, w^{\prime} \in W^{\delta(K)}$ with $w^{\prime} \geqslant_{J, \delta} w$, there exists $x \in W^{\delta(K)}, u \in W_{J}$ and $u_{1} \in W_{K}$, such that $x \geqslant w \delta(u)$ and $w^{\prime}=u_{1}^{-1} u^{-1} x \delta\left(u_{1}\right)$.

Proof. Since $w^{\prime} \geqslant_{J, \delta} w$, there exists $v_{1} \in W_{J}$, such that $w^{\prime} \geqslant v_{1}^{-1} w \delta\left(v_{1}\right)$. By corollary 3.4, there exists $v^{\prime} \leqslant v_{1}$, such that $v^{\prime} w^{\prime} \geqslant w \delta\left(v_{1}\right) \geqslant w \delta\left(v^{\prime}\right)$. Let $v$ be a minimal element in the set $\left\{v \in W_{J} \mid v w^{\prime} \geqslant w \delta(v)\right\}$. Then $l\left(v w^{\prime}\right)=l(v)+l\left(w^{\prime}\right)$. Now assume that $v w^{\prime}=x \delta\left(v^{\prime}\right)$ for some $x \in W^{\delta(K)}$ and $v^{\prime} \in W_{K}$. Then there exists $v_{1}^{\prime} \leqslant v^{\prime}$, such that $x \geqslant w \delta(v) \delta\left(v_{1}^{\prime}\right)^{-1}$. By lemma 3.10, $x \delta\left(v_{1}^{\prime}\right)=v_{2} w^{\prime}$ for some $v_{2} \leqslant v$. Since $l\left(x \delta\left(v_{1}^{\prime}\right)\right)=l(x)+l\left(v_{1}^{\prime}\right), v_{2} w^{\prime}=x \delta\left(v_{1}^{\prime}\right) \geqslant w \delta(v) \geqslant w \delta\left(v_{2}\right)$. Therefore, $v_{2}=v$ and $v_{1}^{\prime}=v^{\prime}$. So $x \geqslant w \delta(v) \delta\left(v^{\prime}\right)^{-1}$. Now set $u=v\left(v^{\prime}\right)^{-1}$ and $u_{1}=v^{\prime}$. Then $w^{\prime}=v^{-1} x \delta\left(v^{\prime}\right)=u_{1}^{-1} u^{-1} x \delta\left(u_{1}\right)$.

3.12. A relation $\leqslant$ is a partial order on a set $S$ if it has:

1. Reflexivity: $a \leqslant a$ for all $a \in S$.

2. Antisymmetry: $a \leqslant b$ and $b \leqslant a$ implies $a=b$.

3. Transitivity: $a \leqslant b$ and $b \leqslant c$ implies $a \leqslant c$.

Proposition 3.13. The relation $\leqslant \delta$ on the set $\mathcal{I}_{\delta}$ is a partial order.

Proof. Reflexivity is clear from the definition.

For $\left(J_{1}, w_{1}\right),\left(J_{2}, w_{2}\right) \in \mathcal{I}_{\delta}$ with $\left(J_{1}, w_{1}\right) \leqslant \delta\left(J_{2}, w_{2}\right)$ and $\left(J_{2}, w_{2}\right) \leqslant \delta\left(J_{1}, w_{1}\right)$, we have that $J_{1}=J_{2}$ and $l\left(w_{1}\right)=l\left(w_{2}\right)$. Since $w_{1} \geqslant w_{2}^{\prime}$ for some $w_{2}^{\prime} \sim_{J, \delta} w_{2}$ and $l\left(w_{1}\right)=l\left(w_{2}\right)=l\left(w_{2}^{\prime}\right), w_{1}=w_{2}^{\prime} \in W^{\delta\left(J_{2}\right)}$. Hence $w_{1}=w_{2}^{\prime}=w_{2}$. Therefore $\left(J_{1}, w_{1}\right)=\left(J_{2}, w_{2}\right)$. Antisymmetry is proved.

Let $\left(J_{1}, w_{1}\right),\left(J_{2}, w_{2}\right)$ and $\left(J_{3}, w_{3}\right) \in \mathcal{I}_{\delta}$. Assume that $\left(J_{1}, w_{1}\right) \leqslant \delta\left(J_{2}, w_{2}\right)$ and $\left(J_{2}, w_{2}\right) \leqslant \delta\left(J_{3}, w_{3}\right)$. Then $J_{1} \subset J_{2} \subset J_{3}$. Moreover, there exists $x \in W^{\delta\left(J_{2}\right)}$, $u \in W_{J_{3}}$ and $u_{1} \in W_{J_{2}}$, such that $x \geqslant w_{3} \delta(u)$ and $w_{2}=u_{1}^{-1} u^{-1} x \delta\left(u_{1}\right)$. Since $w_{1} \geqslant J_{2}, \delta w_{2}$, there exists $u_{2} \in W_{J_{2}}$, such that $w_{1} \geqslant u_{2}^{-1} u^{-1} x \delta\left(u_{2}\right)$. Note that $l\left(x \delta\left(u_{2}\right)\right)=l(x)+l\left(u_{2}\right)$ and $x \geqslant w_{3} \delta(u)$. Thus $x \delta\left(u_{2}\right) \geqslant w_{3} \delta\left(u u_{2}\right)$. By corollary 3.4 , there exists $v \leqslant u u_{2}$, such that $w_{1} \geqslant v^{-1} w_{3} \delta\left(u u_{2}\right)$. By section 3.7, $w_{1} \geqslant_{J_{3}, \delta} w_{3}$. Transitivity is proved. 


\section{The Closure of ANy $G$-Stable Piece}

4.1. We have that

$$
G^{1}=\sqcup_{w \in W} B \dot{w} U^{-} \dot{w}_{0}^{\delta(J)} g_{0}=\sqcup_{w \in W} B \dot{w} \dot{w}_{0}^{\delta(J)} U_{P_{\delta(J)}^{-}} U_{\delta(J)} g_{0}
$$

Moreover, $B \dot{w} U^{-}=\sqcup_{b \in U_{J}^{-} \cap^{-1} U^{-}} B \dot{w} U_{P_{J}^{-}} b$. Thus

$$
\begin{aligned}
B \dot{w} U_{P_{J}^{-}} U_{J} & =B \dot{w} \dot{w}_{0}^{J} U^{-} \dot{w}_{0}^{J}=\sqcup_{b \in U_{J}^{-} \cap^{\left(\dot{w} \dot{w}_{0}^{J}\right)^{-1} U^{-}}} B \dot{w} \dot{w}_{0}^{J} U_{P_{J}^{-}} b \dot{w}_{0}^{J} \\
& =\sqcup_{b \in U_{J} \cap^{\dot{w}^{-1}} U^{-}} B \dot{w} U_{P_{J}^{-}} .
\end{aligned}
$$

Note that if $w=w^{\prime} u$ with $w^{\prime} \in W^{J}$ and $u \in W_{J}$, then

$$
U_{J} \cap \dot{w}^{-1} U^{-}=\dot{u}^{-1}\left({ }^{\dot{u}} U_{J} \cap\left(\dot{w}^{\prime}\right)^{-1} U^{-}\right)=\dot{u}^{-1}\left({ }^{\dot{u}} U_{J} \cap U_{J}^{-}\right)=U_{J} \cap \dot{u}^{-1} U_{J}^{-} .
$$

Lemma 4.2. Let $(J, w) \in \mathcal{I}_{\delta}$. For any $u \in W$ and $b \in B$, there exists $v \leqslant u$, such that $\dot{u} b \dot{w} \in B \dot{v} \dot{w} U_{P_{\delta(J)}^{-}} U_{\delta(J)}$.

Proof. We will prove the statement by induction on $l(u)$.

If $u=1$, then the statement holds. If $u=s_{i} u_{1}$ with $l\left(u_{1}\right)=l(u)-1$, then by induction hypothesis, there exists $v_{1} \leqslant u_{1}$, such that $\dot{u}_{1} b \dot{w} \in b^{\prime} \dot{v}_{1} \dot{w} U_{P_{\delta(J)}^{-}} U_{\delta(J)}$ for some $b^{\prime} \in B$. Write $b^{\prime}=b_{1} b_{2}$, where $b_{1} \in U_{P_{\{i\}}}$ and $b_{2} \in U_{\{i\}}$. Then $\dot{s}_{i} b^{\prime} \dot{v}_{1} \dot{w}=$ $\left(\dot{s}_{i} b_{1} \dot{s}_{i}^{-1}\right) \dot{s}_{i} b_{2} \dot{v}_{1} \dot{w}$ with $\dot{s}_{i} b_{1} \dot{s}_{i}^{-1} \in B$.

If $\left(\dot{v}_{1} \dot{w}\right)^{-1} b_{2} \dot{v}_{1} \dot{w} \in U_{P_{\delta(J)}^{-}} U_{\delta(J)}$, then $\dot{s}_{i} b_{2} \dot{v}_{1} \dot{w} \in \dot{s}_{i} \dot{v}_{1} \dot{w} U_{P_{\delta(J)}^{-}} U_{\delta(J)}$. Otherwise, $b_{2} \neq 1$ and $\left(\dot{v}_{1} \dot{w}\right)^{-1} U_{\{i\}}^{-} \dot{v}_{1} \dot{w} \subset U_{P_{\delta(J)}^{-}} U_{\delta(J)}$. Note that $\dot{s}_{i} b_{2} \in B U_{\{i\}}^{-}$. Thus $\dot{s}_{i} b_{2} \dot{v}_{1} \dot{w} \in B \dot{v}_{1} \dot{w} U_{P_{\delta(J)}^{-}} U_{\delta(J)}$. The statement holds in both cases.

4.3. Let $z \in(G, 1) \cdot h_{J, \delta}$. Then $z$ can be written as $z=\left(b \dot{w} \dot{u} b^{\prime}, 1\right) \cdot h_{J, \delta}$ with $b \in B$, $w \in W^{\delta(J)}, u \in W_{\delta(J)}$ and $b^{\prime} \in U_{\delta(J)} \cap{ }^{\dot{u}^{-1}} U_{\delta(J)}^{-}$. Moreover, $w, u, b^{\prime}$ are uniquely determined by $z$.

Set $J_{0}=J$. To $z \in(G, 1) \cdot h_{J, \delta}$, we associate a sequence $\left(J_{i}, w_{i}, v_{i}, v_{i}^{\prime}, c_{i}, z_{i}\right)_{i \geqslant 1}$ with $J_{i} \subset J, w_{i} \in W^{\delta(J)}, v_{i} \in W_{J_{i-1}} \cap{ }^{J_{i}} W, v_{i}^{\prime} \in W_{J_{i}}, c_{i} \in U_{\delta\left(J_{i-1}\right)} \cap{ }^{\dot{\delta}\left(v_{i}^{-1}\right)} U_{\delta\left(J_{i-1}\right)}^{-}$ and $z_{i} \in\left(B \dot{w}_{i} \dot{\delta}\left(v_{i}^{\prime}\right) U_{\delta(J)} \dot{\delta}\left(v_{i}\right) c_{i}, 1\right) \cdot h_{J, \delta}$ and in the same $G_{d i a g}$-orbit as $z$. The sequence is defined as follows.

Assume that $z \in\left(B \dot{w} \dot{\delta}(u) U_{\delta(J)}, 1\right) \cdot h_{J, \delta}$ with $w \in W^{\delta(J)}$ and $u \in W_{\delta(J)}$. Then set $z_{1}=z, J_{1}=J, w_{1}=w, v_{1}=1, v_{1}^{\prime}=u$ and $c_{1}=1$.

Assume that $k \geqslant 1$, that $J_{k}, w_{k}, v_{k}, v_{k}^{\prime}, c_{k}, z_{k}$ are already defined and that $J_{k} \subset$ $J_{k-1}, w_{k} \in W^{\delta(J)}, W_{J_{k-1}} w_{k} \subset W^{\delta(J)} W_{\delta\left(J_{k}\right)}, v_{k} \in W_{J_{k-1}} \cap{ }^{J_{k}} W, v_{k}^{\prime} \in W_{J_{k}}$, $c_{k} \in U_{\delta\left(J_{k-1}\right)} \cap \dot{\delta}\left(v_{k}^{-1}\right) U_{\delta\left(J_{k-1}\right)}^{-}$and $z_{k} \in\left(B \dot{w}_{k} \dot{\delta}\left(v_{k}^{\prime}\right) U_{\delta(J)} \dot{\delta}\left(v_{k}\right) c_{k}, 1\right) \cdot h_{J, \delta}$. Set $z_{k+1}=$ $\left(g_{0}^{-1} \dot{\delta}\left(v_{k}\right) c_{k} g_{0}, g_{0}^{-1} \dot{\delta}\left(v_{k}\right) c_{k} g_{0}\right) z_{k}$. Then $z_{k+1} \in(G, 1) \cdot h_{J, \delta}$. Moreover, by lemma 4.2, there exists $x_{k} \leqslant v_{k}$, such that $z_{k+1} \in\left(B \dot{x}_{k} w_{k} \dot{\delta}\left(v_{k}^{\prime}\right) U_{\delta(J)}, 1\right) \cdot h_{J, \delta}$.

Let $y_{k+1}$ be the unique element of the minimal length in $W_{J_{k}} x_{k} w_{k} \delta\left(v_{k}^{\prime}\right) W_{\delta(J)}$. Set $J_{k+1}=J_{k} \cap \delta^{-1} \operatorname{Ad}\left(y_{k+1}^{-1}\right) J_{k}$. Since $W_{J_{k-1}} w_{k} \subset W^{\delta(J)} W_{\delta\left(J_{k}\right)}$, then we have that $x_{k} w_{k} \delta\left(v_{k}^{\prime}\right)=w_{k+1} \delta\left(v_{k+1}^{\prime} v_{k+1}\right)$ for some $w_{k+1} \in W^{\delta(J)}, v_{k+1}^{\prime} \in W_{J_{k+1}}$ and $v_{k+1} \in W_{J_{k}} \cap J_{k+1}^{J_{k}} W$. Note that $W_{J_{k}} w_{k+1} \subset W^{\delta(J)} W_{\delta(J) \cap \operatorname{Ad}\left(y_{k+1}^{-1}\right) J_{k}}$. On the other hand, $W_{J_{k}} w_{k+1} \subset W_{J_{k-1}} w_{k} W_{\delta\left(J_{k}\right)} \subset W^{\delta(J)} W_{\delta\left(J_{k}\right)}$. Thus $W_{J_{k}} w_{k+1} \subset$ $\left(W^{\delta(J)} W_{\delta(J) \cap \operatorname{Ad}\left(y_{k+1}^{-1}\right) J_{k}}\right) \cap\left(W^{\delta(J)} W_{\delta\left(J_{k}\right)}\right)=W^{\delta(J)} W_{\delta\left(J_{k+1}\right)}$. 
Moreover $z_{k+1} \in\left(B \dot{w}_{k+1} \dot{\delta}\left(v_{k+1}^{\prime}\right) U_{\delta(J)} \dot{\delta}\left(v_{k+1}\right) c_{k+1}, 1\right) \cdot h_{J, \delta}$ for a unique $c_{k+1} \in$ $U_{J_{k}} \cap \dot{\delta}\left(v_{k+1}^{-1}\right) U_{J_{k}}^{-}$.

This completes the inductive definition. Moreover, for sufficient large $n$, we have that $J_{n}=J_{n+1}=\cdots, w_{n}=w_{n+1}=\cdots, v_{n}^{\prime}=v_{n+1}^{\prime}=\cdots$ and $v_{n}=v_{n+1}=\cdots=1$.

4.4. Let $K \subset J, y \in{ }^{K} W^{\delta(K)}$ and $K=\operatorname{Ad}(y) \delta(K)$. Then for any $u \in W_{K}$, we have that

$$
\left(B \dot{y} \dot{\delta}(u) U_{\delta(K)}, 1\right) \cdot h_{J, \delta} \subset G_{\text {diag }}\left(\dot{y} L_{\delta(K)}, B\right) \cdot h_{J, \delta}=G_{\text {diag }}\left(\dot{y} L_{\delta(K)}, U_{P_{K}}\right) \cdot h_{J, \delta} .
$$

For any $l \in L_{K}$, there exists $l^{\prime} \in L_{K}$, such that $l^{\prime} \dot{y} g_{0} l\left(l^{\prime}\right)^{-1} \in \dot{y} g_{0}\left(L_{K} \cap B\right)$. Thus $\left(L_{K}\right)_{\text {diag }}\left(\dot{y}\left(L_{\delta(K)} \cap B\right), U_{P_{K}}\right) \cdot h_{J, \delta}=\left(\dot{y} L_{\delta(K)}, U_{P_{K}}\right) \cdot h_{J, \delta}$. Hence

$\left(B \dot{y} \dot{\delta}(u) U_{\delta(K)}, 1\right) \cdot h_{J, \delta} \subset G_{\text {diag }}\left(\dot{y}\left(L_{\delta(K)} \cap B\right), U_{P_{K}}\right) \cdot h_{J, \delta}=G_{\text {diag }}(\dot{y}, B) \cdot h_{J, \delta}=Z_{J, \delta}^{y}$.

Now for any $z \in(G, 1) \cdot h_{J, \delta}$, let $\left(z_{i}, J_{i}, w_{i}, v_{i}, v_{i}^{\prime}, c_{i}\right)_{i \geqslant 1}$ be the sequence associated to $z$. Assume that $J_{n}=J_{n+1}=\cdots, w_{n}=w_{n+1}=\cdots, v_{n}^{\prime}=v_{n+1}^{\prime}=\cdots$ and $v_{n}=v_{n+1}=\cdots=1$. Then we have showed that $z_{n} \in Z_{J, \delta}^{w_{n}}$. Thus $z \in Z_{J, \delta}^{w_{n}}$.

Note that for any $z \in Z_{J, \delta}, z$ is in the same $G$-orbit as an element of the form $(G, 1) \cdot h_{J, \delta}$. Therefore, given $z \in Z_{J, \delta}$, our procedure determines the $G$-stable piece $Z_{J, \delta}^{w}$ that contains $z$.

Now we are able to describe the closure of $Z_{J, \delta}^{w}$. In theorem 4.5 , we will only consider subvarieties of $\bar{G}^{1}$ and for any subvariety $X$ of $\bar{G}^{1}$, we denote by $\bar{X}$ the closure of $X$ in $\bar{G}^{1}$.

Theorem 4.5. For any $(J, w) \in \mathcal{I}_{\delta}$, we have that

$$
\overline{Z_{J, \delta}^{w}}=\sqcup_{\left(K, w^{\prime}\right) \leqslant \delta}(J, w) Z_{K, \delta}^{w^{\prime}} .
$$

Proof. Define $\pi^{\prime}: G \times \overline{[I, 1,1]_{\delta}} \rightarrow \bar{G}^{1}$ by $\pi(g, z)=(g, g) \cdot z$. The morphism is invariant under the $B$-action defined by $b(g, z)=\left(g b^{-1}, \pi^{\prime}(b, z)\right)$. Denote by $G \times{ }_{B}$ $\overline{[I, 1,1]_{\delta}}$ the quotient; we obtain a morphism $\pi: G \times_{B} \overline{[I, 1,1]_{\delta}} \rightarrow \bar{G}^{1}$. Because $G / B$ is projective, $\pi$ is proper and hence surjective.

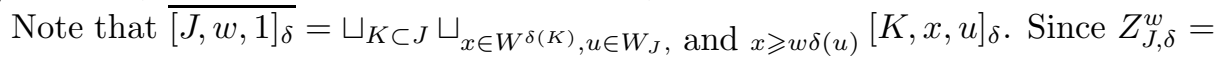
$\pi\left(G \times{ }_{B}[J, w, 1]_{\delta}\right)$, we have that

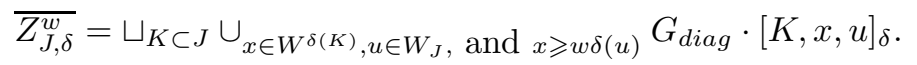

For any $z \in[K, x, u]_{\delta}$ with $x \in W^{\delta(K)}, u \in W_{J}$ and $x \geqslant w \delta(u)$, we have that $z \in(B \dot{x}, B \dot{u}) \cdot h_{K, \delta}=G_{\text {diag }}\left(\dot{u}^{-1} B \dot{x}, 1\right) \cdot h_{K, \delta} \subset \sqcup_{v \leqslant u^{-1}} G_{\text {diag }}\left(B \dot{v} \dot{x} U_{\delta(K)}, 1\right) \cdot h_{K, \delta}$.

Fix $v \leqslant u^{-1}$ and $z^{\prime} \in\left(B \dot{v} \dot{x} U_{\delta(K)}, 1\right) \cdot h_{K, \delta}$. Let $\left(z_{i}, J_{i}, w_{i}, v_{i}, v_{i}^{\prime}, c_{i}\right)_{i \geqslant 1}$ be the sequence associated to $z^{\prime}$. Then for any $i$, there exists $x_{i} \leqslant v_{i}$, such that $x_{i} w_{i} \delta\left(v_{i}^{\prime}\right)=$ $w_{i+1} \delta\left(v_{i+1}^{\prime} v_{i+1}\right)$. Assume that $J_{n}=J_{n+1}=\cdots, w_{n}=w_{n+1}=\cdots, v_{n}^{\prime}=v_{n+1}^{\prime}=$ $\cdots$ and $v_{n}=v_{n+1}=\cdots=1$. Set $x_{\infty}=x_{n} x_{n-1} \cdots x_{2}$ and $v_{\infty}=v_{n}^{\prime}\left(v_{n} v_{n-1} \cdots v_{2}\right)$. Note that $x_{1}=v_{1}=1$. Then $x_{\infty} v x=x_{\infty} w_{1} \delta\left(v_{1}^{\prime}\right)=w_{n} \delta\left(v_{\infty}\right)$. Since $v_{n}^{\prime} \in W_{J_{n+1}}$ and $v_{i} \in W_{J_{i}} \cap{ }^{J_{i+1}} W$, we have that $l\left(v_{\infty}\right)=l\left(v_{n}^{\prime}\right)+l\left(v_{n}\right)+l\left(v_{n-1}\right)+\cdots+l\left(v_{2}\right)$. Thus $x_{\infty} \leqslant v_{\infty}$. By section 4.4, $z^{\prime} \in Z_{K, x_{\infty} v x \delta\left(v_{\infty}^{-1}\right)}$. Note that $v^{-1} \leqslant u$ and $l(w u)=$ $l(w)+l(u)$. Thus $w \delta\left(v^{-1}\right) \leqslant w \delta(u) \leqslant x$. Similarly, $w \delta\left(v^{-1} x_{\infty}^{-1}\right) \leqslant x \delta\left(v_{\infty}^{-1}\right)$. By corollary 3.4, there exist $v^{\prime} \leqslant v^{-1} x_{\infty}^{-1}$, such that $\left(v^{\prime}\right)^{-1} w \delta\left(v^{-1} x_{\infty}^{-1}\right) \leqslant x_{\infty} v x \delta\left(v_{\infty}^{-1}\right)$. Thus by proposition $3.8, x_{\infty} v x \delta\left(v_{\infty}^{-1}\right) \geqslant{ }_{J, \delta} w$.

For any $K \subset J$ and $w^{\prime} \in W^{\delta(K)}$ with $w^{\prime} \geqslant J, \delta w$, there exists $x \in W^{\delta(K)}, u \in W_{J}$ and $u_{1} \in W_{K}$, such that $x \geqslant w \delta(u)$ and $w^{\prime}=u_{1}^{-1} u^{-1} x \delta\left(u_{1}\right)$. Since $[K, x, u]_{\delta} \subset$ 
$\overline{[J, w, 1]_{\delta}}$. We have that $(\dot{x} T, \dot{u}) \cdot h_{K, \delta} \subset \overline{Z_{J, \delta}^{w}}$. Therefore $\left(\dot{u}^{-1} \dot{x} T, 1\right) \cdot h_{K, \delta} \subset \overline{Z_{J, \delta}^{w}}$. Note that $u^{-1} x=u_{1} w^{\prime} \delta\left(u_{1}\right)^{-1}$. Then $\left(\dot{u}_{1} \dot{w}^{\prime} \dot{\delta}\left(u_{1}\right)^{-1} T, 1\right) \cdot h_{K, \delta} \subset \overline{Z_{J, \delta}^{w}}$. Thus

$$
\left(\dot{u}_{1}^{-1}, \dot{u}_{1}^{-1}\right)\left(\dot{u}_{1} \dot{w}^{\prime} \dot{\delta}\left(u_{1}\right)^{-1} T, 1\right) \cdot h_{K, \delta}=\left(\dot{w}^{\prime} T, 1\right) \cdot h_{K, \delta} \subset \overline{Z_{J, \delta}^{w}} .
$$

By proposition 1.12, $Z_{K, \delta}^{w^{\prime}} \subset \overline{Z_{J, \delta}^{w}}$. The theorem is proved.

Our method also works in another situation.

Proposition 4.6. The closure of $Z_{J, 1, \delta}^{w}$ in $Z_{J, 1, \delta}$ is $\sqcup_{w^{\prime} \in W^{\delta(J)}, w \geqslant{ }_{J} w^{\prime}} Z_{J, 1, \delta}^{w^{\prime}}$.

Proof. In the proof, we will only consider subvarieties of $Z_{J, 1, \delta}$, and for any subvariety $X$ of $Z_{J, 1, \delta}$, we denote by $\bar{X}$ its closure in $Z_{J, 1, \delta}$.

Note that the morphism $\pi: Z_{J, 1, \delta} \rightarrow \mathcal{P}_{J}$, defined by $\pi(P, Q, \gamma)=P$ for $(P, Q, \gamma) \in Z_{J, 1, \delta}$ is a locally trivial fibration with isomorphic fibers. Moreover, $i: \pi^{-1}\left(P_{J}\right) \rightarrow G^{1} / H_{P_{J}}$, defined by $i(P, Q, \gamma)=\gamma$ for $(P, Q, \gamma) \in \pi^{-1}\left(P_{J}\right)$, is an isomorphism. Now $[J, w, 1]_{1, \delta} \subset \pi^{-1}\left(P_{J}\right)$ and $i\left([J, w, 1]_{1, \delta}\right)=B \dot{w} B g_{0} / H_{P_{J}}$. Thus $\overline{[J, w, 1]_{1, \delta}}=\sqcup_{w^{\prime} \leqslant w}\left[J, w^{\prime}, 1\right]_{1, \delta}$. For any $w^{\prime} \in W^{J}$ with $w \geqslant_{J, \delta} w^{\prime}$, there exists $u \in$ $W_{J}$, such that $w \geqslant u^{-1} w^{\prime} \delta(u)$. Thus $\left(\dot{u}^{-1} \dot{w}^{\prime} \dot{\delta}(u) T, 1\right) \cdot h_{J, 1, \delta} \subset \overline{[J, w, 1]_{1, \delta}}$. Hence $G_{\text {diag }}\left(\dot{u}^{-1} \dot{w}^{\prime} \dot{\delta}(u) T, 1\right) \cdot h_{J, 1, \delta}=G_{\text {diag }}\left(\dot{w}^{\prime} T, 1\right) \cdot h_{J, 1, \delta} \subset \overline{Z_{J, 1, \delta}^{w}}$. So $Z_{J, 1, \delta}^{w^{\prime}} \subset \overline{Z_{J, 1, \delta}^{w}}$.

On the other hand, for any $z \in\left[J, w^{\prime}, 1\right]_{1, \delta}$, by the similar argument as we did in sections 4.3 and 4.4 , there exists $u \leqslant v \in W_{J}$, such that $u w^{\prime} \delta\left(v^{-1}\right) \in W^{\delta(J)}$ and $z \in Z_{J, 1, \delta}^{u w^{\prime} v^{-1}}$. If moreover, $w^{\prime} \leqslant w$, then $w \geqslant u^{-1}\left(u w^{\prime} \delta\left(v^{-1}\right)\right) \delta(v)$. Thus $w \geqslant_{J, \delta}$ $u w^{\prime} v^{-1}$. Therefore $z \in \sqcup_{w^{\prime} \in W^{\delta(J)}, w \geqslant J, \delta} w^{\prime} Z_{J, 1, \delta}^{w^{\prime}}$. The proposition is proved.

\section{The Cellular DeCOMPosition}

5.1. A finite partition of a variety $X$ into subsets is said to be an $\alpha$-partition if the subsets in the partition can be indexed $X_{1}, X_{2}, \ldots, X_{n}$ in such a way that $X_{1} \cup X_{2} \cup \cdots \cup X_{i}$ is closed in $X$ for $i=1,2, \ldots, n$. We say that a variety has a cellular decomposition if it admits an $\alpha$-partition into subvarieties which are affine spaces. It is easy to see that if a variety $X$ admits an $\alpha$-partition into subvarieties and each subvariety has a cellular decomposition, then $X$ has a cellular decomposition.

Lemma 5.2. Let $(J, w) \in \mathcal{I}_{\delta}, K \subset J$ and $w^{\prime} \in W$ with $\operatorname{Ad}\left(w^{\prime}\right) \delta(K)=K$. If $w^{\prime} v \geqslant_{J, \delta} w$ for some $v \in W_{\delta(K)}$, then $w^{\prime} \geqslant_{J, \delta} w$.

Proof. Fix $w^{\prime}$ and $(J, w)$. It suffices to prove the following statement:

Let $u \in W_{J}$ and $v \in W_{\delta(K)}$. If $w^{\prime} v \geqslant u^{-1} w \delta(u)$, then $w^{\prime} \geqslant_{J, \delta} w$.

We argue by induction on $l(u)$. Assume that the statement holds for all $u^{\prime}<u$. Then I will prove that the statement holds for $u$ by induction on $l(v)$. If $l(v)=0$, then $v=1$ and the statement holds in this case. Now assume that $l(v)>0$.

Set $u=u_{1} u_{2}$ with $u_{1} \in W^{K}$ and $u_{2} \in W_{K}$. If $u_{2}=1$, then $u \in W^{K}$ and $w \delta(u) \in W^{\delta(K)}$. By corollary 3.4, there exists $u^{\prime} \leqslant u$, such that $u^{\prime} w^{\prime} v \geqslant w \delta(u)$. Assume that $v=v^{\prime} s_{k}$ for $v^{\prime}<v$ and $k \in \delta(K)$. Then $w \delta(u)<w \delta(u) s_{k}$. By section 3.1, $w \delta(u) \leqslant u^{\prime} w^{\prime} v^{\prime}$. By corollary 3.4, there exists $u_{1}^{\prime} \leqslant u^{\prime} \leqslant u$, such that $w^{\prime} v^{\prime} \geqslant\left(u_{1}^{\prime}\right)^{-1} w \delta(u)$. By the remark of proposition 3.8, $w^{\prime} v^{\prime} \geqslant\left(u_{2}^{\prime}\right)^{-1} w \delta\left(u_{2}^{\prime}\right)$ for some $u_{2}^{\prime} \leqslant u_{1}^{\prime}$. Thus by induction hypothesis, $w^{\prime} \geqslant_{J, \delta} w$.

If $u_{2} \neq 1$, then $l\left(u_{1}\right)<l(u)$. By corollary 3.4 , there exists $u_{3} \leqslant u_{2}$ and $u_{4} \leqslant u_{2}^{-1}$, such that $u_{3} w^{\prime} v \delta\left(u_{4}\right) \geqslant u_{1}^{-1} w \delta\left(u_{1}\right)$. Note that $u_{3} w^{\prime} v u_{4}=w^{\prime}\left(\left(w^{\prime}\right)^{-1} u_{3} w^{\prime}\right) v \delta\left(u_{4}\right) \in$ $w^{\prime} W_{\delta(K)}$. By induction hypothesis on $l\left(u_{1}\right), w^{\prime} \geqslant_{J, \delta} w$. 
5.3. Let $J \subset I$. For $w \in W$, set

$$
\begin{aligned}
& I_{1}(J, w, \delta)=\max \left\{K \subset J \mid w \in W^{\delta(K)}\right\}, \\
& I_{2}(J, w, \delta)=\max \left\{K \subset J \mid \operatorname{Ad}(w) \Phi_{\delta(K)}=\Phi_{K}\right\} .
\end{aligned}
$$

Now let $(J, w) \in \mathcal{I}_{\delta}$. Set

$$
W_{\delta}(J, w)=\left\{u \in W \mid u \geqslant_{J, \delta} w, I_{2}(J, u, \delta) \subset I_{1}(J, u, \delta)\right\} .
$$

For any $u \in W_{\delta}(J, w)$, set

$$
\begin{aligned}
X_{u}^{(J, w, \delta)} & =\sqcup_{K \subset I_{1}(J, u, \delta)} \sqcup_{v \in W_{\delta\left(I_{2}(J, u, \delta)\right)} \cap W^{\delta(K)}} Z_{K, \delta}^{u v} \\
& =\sqcup_{v \in W_{\delta\left(I_{2}(J, u, \delta)\right)}} \sqcup_{K \subset I_{1}(J, u v, \delta)} Z_{K, \delta}^{u v} .
\end{aligned}
$$

For $w^{\prime} \geqslant_{J, \delta} w$, we have that $w^{\prime}=u v$ for some $u \in W^{\delta\left(I_{2}\left(J, w^{\prime}\right)\right)}$ and $v \in$ $W_{\delta\left(I_{2}\left(J, w^{\prime}\right)\right)}$. Then $I_{2}(J, u, \delta)=I_{2}\left(J, w^{\prime}, \delta\right) \subset I_{1}(J, u, \delta)$. By lemma $5.2, u \geqslant_{J, \delta} w$. Thus $u \in W_{\delta}(J, w)$ and $\sqcup_{K \subset I_{1}\left(J, w^{\prime}, \delta\right)} Z_{K, \delta}^{w^{\prime}} \subset X_{u}^{(J, w, \delta)}$.

For $u_{1}, u_{2} \in W(J, w)$ and $v_{1} \in W_{\delta\left(I_{2}\left(J, u_{1}\right)\right)}, v_{2} \in W_{\delta\left(I_{2}\left(J, u_{2}\right)\right)}$ with $u_{1} v_{1}=u_{2} v_{2}$, we have that $I_{2}\left(J, u_{1}, \delta\right)=I_{2}\left(J, u_{1} v_{1}, \delta\right)=I_{2}\left(J, u_{2} v_{2}, \delta\right)=I_{2}\left(J, u_{2}, \delta\right)$. Note that $u_{1}, u_{2} \in W^{\delta\left(I_{2}\left(J, u_{1}\right)\right)}$. Thus $u_{1}=u_{2}$ and $v_{1}=v_{2}$.

Therefore $\overline{Z_{J, \delta}^{w}}=\sqcup_{u \in W_{\delta}(J, w)} X_{u}^{(J, w, \delta)}$.

Lemma 5.4. Let $(J, w) \in \mathcal{I}_{\delta}$. Set $I_{2}=I_{2}(J, w, \delta)$. For $K \subset J$, we have that

$$
\sqcup_{v \in W_{\delta\left(I_{2}\right)} \cap W^{\delta(K)}}\left(L_{I_{2}}\right)_{d i a g}\left(\dot{w} \dot{v}, B \cap L_{I_{2}}\right) \cdot h_{K, \delta}=\left(L_{I_{2}}, L_{I_{2}}\right)(\dot{w}, 1) \cdot h_{K, \delta} .
$$

Proof. At first, we will prove the case when $K \subset I_{2}$. In this case, set $g_{1}=$ $g_{0} \dot{w}$. Then $g_{1} L_{\delta\left(I_{2}\right)} g_{1}^{-1}=L_{\delta\left(I_{2}\right)}$ and $g_{1}\left(L_{\delta\left(I_{2}\right)} \cap B\right) g_{1}^{-1}=L_{\delta\left(I_{2}\right)} \cap B$. Now consider $\overline{L_{\delta\left(I_{2}\right)} / Z\left(L_{\delta\left(I_{2}\right)}\right)} g_{1}$ (a variety that is isomorphic to $\overline{L_{\delta\left(I_{2}\right)} / Z\left(L_{\delta\left(I_{2}\right)}\right)}$, but with "twisted" $L_{\delta\left(I_{2}\right)} \times L_{\delta\left(I_{2}\right)}$ action; see section 2.3). We have that

$$
\sqcup_{v \in W_{\delta\left(I_{2}\right)} \cap W^{\delta(K)}}\left(L_{\delta\left(I_{2}\right)}\right)_{\text {diag }}\left(\dot{v}, B \cap L_{\delta\left(I_{2}\right)}\right) \cdot\left(h_{\delta(K)} g_{1}\right)=\left(L_{\delta\left(I_{2}\right)}, L_{\delta\left(I_{2}\right)}\right) \cdot\left(h_{\delta(K)} g_{1}\right) \text {. }
$$

(In the case when $g_{1}^{n} \in L_{\delta\left(I_{2}\right)}$ for some $n \in \mathbf{N}, L_{\delta\left(I_{2}\right)} g_{1}$ is a connected component of the group generated by $L_{\delta\left(I_{2}\right)}$ and $g_{1}$. In this case, the left hand side is the union of some $L_{\delta\left(I_{2}\right)}$-stable pieces and the equality follows from [L4, 12, 3]. The general case can be shown in the same way.)

Therefore

$$
\begin{aligned}
& \sqcup_{v \in W_{\delta\left(I_{2}\right)} \cap W^{\delta(K)}}\left(\dot{w}^{-1}, g_{0}\right)\left(L_{I_{2}}\right)_{\text {diag }}\left(\dot{w}, g_{0}^{-1}\right)\left(\dot{v}, B \cap L_{\delta\left(I_{2}\right)}\right) \cdot h_{\delta(K)} \\
& =\sqcup_{v \in W_{\delta\left(I_{2}\right)} \cap W^{\delta(K)}}\left(1, g_{1}\right)\left(L_{\delta\left(I_{2}\right)}\right)_{\operatorname{diag}}\left(1, g_{1}^{-1}\right)\left(\dot{v}, B \cap L_{\delta\left(I_{2}\right)}\right) \cdot h_{\delta(K)} \\
& =\left(L_{\delta\left(I_{2}\right)}, L_{\delta\left(I_{2}\right)}\right)(\dot{w}, 1) \cdot h_{\delta(K)} .
\end{aligned}
$$

Note that $h_{K, \delta}=h_{\delta(K)} g_{0}$. Then $\sqcup_{v \in W_{\delta\left(I_{2}\right)} \cap W^{\delta(K)}}\left(\dot{w}^{-1}, 1\right)\left(L_{I_{2}}\right)_{d i a g}\left(\dot{w} \dot{v}, B \cap L_{I_{2}}\right)$. $h_{K, \delta}=\left(L_{\delta\left(I_{2}\right)}, L_{I_{2}}\right) \cdot h_{K, \delta}$. Hence $\sqcup_{v \in W_{\delta\left(I_{2}\right)} \cap W^{\delta(K)}}\left(L_{I_{2}}\right)_{\text {diag }}\left(\dot{w} \dot{v}, B \cap L_{I_{2}}\right) \cdot h_{K, \delta}=$ $\left(L_{I_{2}}, L_{I_{2}}\right) \cdot h_{K, \delta}$.

In the general case, Consider $\pi:\left(L_{\delta\left(I_{2}\right)}, L_{I_{2}}\right) \cdot h_{K, \delta} \rightarrow \overline{L_{\delta\left(I_{2}\right)} / Z\left(L_{\delta\left(I_{2}\right)}\right)} g_{0}$ defined by $\pi\left(\left(l_{1}, l_{2}\right) h_{K, \delta}\right)=\left(l_{1}, l_{2}\right) \cdot\left(h_{\delta(K) \cap \delta\left(I_{2}\right)} g_{0}\right)$ for $l_{1} \in L_{\delta\left(I_{2}\right)}, l_{2} \in L_{I_{2}}$. Here $h_{\delta(K) \cap \delta\left(I_{2}\right)}$ on the right side is the base point in $\overline{L_{\delta\left(I_{2}\right)} / Z\left(L_{\delta\left(I_{2}\right)}\right)}$ that corresponds to $\delta(K) \cap$ $\delta\left(I_{2}\right)$. It is easy to see that the morphism is well-defined. Now define the $T$-action on $\left(L_{\delta\left(I_{2}\right)}, L_{I_{2}}\right) \cdot h_{K, \delta}$ by $t \cdot\left(\left(l_{1}, l_{2}\right) h_{K, \delta}\right)=\left(t l_{1}, l_{2}\right) h_{K, \delta}$ for $t \in T$ and $l_{1} \in L_{\delta\left(I_{2}\right)}, l_{2} \in$ 
$L_{I_{2}}$. Then $T$ acts transitively on $\pi^{-1}(a)$ for any $a \in\left(L_{\delta\left(I_{2}\right)}, L_{I_{2}}\right) \cdot\left(h_{\delta(K) \cap \delta\left(I_{2}\right)} g_{0}\right)$. Now

$$
\begin{aligned}
& \sqcup_{v \in W_{\delta\left(I_{2}\right)} \cap W^{\delta(K)}} \pi\left(\left(\dot{w}^{-1}, 1\right)\left(L_{I_{2}}\right)_{d i a g} \cdot\left(\dot{w} \dot{v}, B \cap L_{I_{2}}\right) \cdot h_{K, \delta}\right) \\
& =\sqcup_{v \in W_{\delta\left(I_{2}\right)} \cap W^{\delta(K)}}\left(\dot{w}^{-1}, 1\right)\left(L_{I_{2}}\right)_{d i a g}\left(\dot{w} \dot{v}, B \cap L_{I_{2}}\right) \cdot\left(h_{\delta(K) \cap \delta\left(I_{2}\right)} g_{0}\right) \\
& =\left(L_{\delta\left(I_{2}\right)}, L_{I_{2}}\right) \cdot\left(h_{\delta(K) \cap \delta\left(I_{2}\right)} g_{0}\right) .
\end{aligned}
$$

Moreover $\sqcup_{v \in W_{\delta\left(I_{2}\right)} \cap W^{\delta(K)}}\left(\dot{w}^{-1}, 1\right)\left(L_{I_{2}}\right)_{\text {diag }} \cdot\left(\dot{w} \dot{v}, B \cap L_{I_{2}}\right) \cdot h_{K, \delta}$ is stable under $T$ action. Thus $\sqcup_{v \in W_{\delta\left(I_{2}\right)} \cap W^{\delta(K)}}\left(\dot{w}^{-1}, 1\right)\left(L_{I_{2}}\right)_{d i a g} \cdot\left(\dot{w} \dot{v}, B \cap L_{I_{2}}\right) \cdot h_{K, \delta}=\left(L_{\delta\left(I_{2}\right)}, L_{I_{2}}\right)$. $h_{K, \delta}$. The lemma is proved.

Proposition 5.5. Let $(J, w) \in \mathcal{I}_{\delta}$ and $u \in W_{\delta}(J, w)$. Set $I_{1}=I_{1}(J, u, \delta), I_{2}=$ $I_{2}(J, u, \delta)$ and $L_{u}^{(J, w, \delta)}=\sqcup_{K \subset I_{1}}\left(L_{I_{2}}, L_{I_{2}}\right)(\dot{u}, 1) \cdot h_{K, \delta}$. Then we have that

(1) $L_{u}^{(J, w, \delta)}$ is a fibre bundle over $\overline{L_{I_{2}} / Z\left(L_{I_{2}}\right)}$ with fibres isomorphic to an affine space of dimension $\left|I_{1}\right|-\left|I_{2}\right|$.

(2) $X_{u}^{(J, w, \delta)}=G_{\text {diag }} \cdot L_{u}^{(J, w, \delta)}$ is isomorphic to $G \times_{P_{I_{2}}}\left(\left(P_{I_{2}}\right)_{\text {diag }} \cdot L_{u}^{(J, w, \delta)}\right)$.

(3) $\left(P_{I_{2}}\right)_{d i a g} \cdot L_{u}^{(J, w, \delta)}=(B \times B) \cdot L_{u}^{(J, w, \delta)} \cong\left(U \cap \dot{w}_{0}^{I_{2}} \dot{u} \dot{w}_{0} U^{-}\right) \times L_{u}^{(J, w, \delta)}$.

Proof. For part (1), note that $L_{u}^{(J, u, \delta)}=\sqcup_{K \subset I_{1}}(\dot{u}, 1)\left(L_{\delta\left(I_{2}\right)}, L_{I_{2}}\right) \cdot h_{K, \delta}=\left(\dot{u} L_{\delta\left(I_{2}\right)}\right.$, $\left.L_{I_{2}}\right) \cdot\left(\sqcup_{K \subset I_{1}}(T, 1) h_{K, \delta}\right)$ is a variety. Consider the morphism

$$
\pi^{\prime}: \sqcup_{K \subset I_{1}}\left(L_{\delta\left(I_{2}\right)}, L_{I_{2}}\right) \cdot h_{K, \delta} \rightarrow \overline{L_{\delta\left(I_{2}\right)} / Z\left(L_{\delta\left(I_{2}\right)}\right)} g_{0}
$$

defined by $\pi^{\prime}\left(\left(l_{1}, l_{2}\right) h_{K, \delta}\right)=\left(l_{1}, l_{2}\right) \cdot\left(h_{\delta(K) \cap \delta\left(I_{2}\right)} g_{0}\right)$ for $l_{1} \in L_{\delta\left(I_{2}\right)}, l_{2} \in L_{I_{2}}$. It is easy to see that $\pi^{\prime}$ is well defined and is a locally trivial fibration with fibers isomorphic to an affine space of dimension $\left|I_{1}\right|-\left|I_{2}\right|$.

Let $v \in W_{\delta\left(I_{2}\right)}$. For $K \subset J$, if $\operatorname{Ad}(u v) \delta(K)=K$, then

$$
\operatorname{Ad}(u) \Phi_{\delta(K)}=\operatorname{Ad}\left(u v^{-1} u^{-1}\right) \Phi_{K} .
$$

Since $u v^{-1} u^{-1} \in W_{I_{2}}$, we have that $\operatorname{Ad}(u) \Phi_{\delta(K)} \subset \Phi_{K \cup I_{2}}$. Thus $\operatorname{Ad}(u) \Phi_{\delta\left(K \cup I_{2}\right)} \subset$ $\Phi_{K \cup I_{2}}$. By the maximal property of $I_{2}, K \cup I_{2} \subset I_{2}$. Thus $I_{2}(J, u v, \delta) \subset I_{2}$. Therefore,

$$
\begin{aligned}
G_{\text {diag }} \cdot L_{u}^{(J, w, \delta)} & =G_{\text {diag }}\left(\sqcup_{K \subset I_{1}}\left(L_{I_{2}}, L_{I_{2}}\right)(\dot{u}, 1) \cdot h_{K, \delta}\right) \\
& =G_{\text {diag }}\left(\sqcup_{K \subset I_{1}} \sqcup_{v \in W_{\delta\left(I_{2}\right)} \cap W^{\delta(K)}}\left(L_{I_{2}}\right)_{\text {diag }}\left(\dot{u} \dot{v}, B \cap L_{I_{2}(J, u, \delta)}\right) \cdot h_{K, \delta}\right) \\
& =\sqcup_{K \subset I_{1}} \sqcup_{v \in W_{\delta\left(I_{2}\right)} \cap W^{\delta(K)}} G_{\text {diag }}\left(\dot{u} \dot{v}, B \cap L_{I_{2}}\right) \cdot h_{K, \delta} \\
& =\sqcup_{K \subset I_{1}} \sqcup_{v \in W_{\delta\left(I_{2}\right)}} Z_{J, \delta}^{u v}=X_{u}^{(J, w, \delta)} .
\end{aligned}
$$

Assume that $(g, g) a=b$ for some $g \in G$ and $a, b \in L_{u}^{(J, u, \delta)}$. Then $a, b$ are in the same $G$ orbit. Note that any element in $L_{u}^{(J, u, \delta)}$ is conjugate by $L_{I_{2}}$ to an element of the form $(\dot{u} \dot{v}, l) h_{K, \delta}$ with $v \in W_{\delta\left(I_{2}\right)}, K \subset I_{1}(J, u v, \delta)$ and $l \in L_{I_{2}} \cap B$. Moreover, $\left(\dot{u} \dot{v}, L_{I_{2}} \cap B\right) \cdot h_{K, \delta} \subset Z_{K, \delta}^{u v}$. Thus if $v_{1} \neq v_{2}$ or $K_{1} \neq K_{2}$, then for any $l, l^{\prime} \in L_{I_{2}} \cap B,\left(\dot{u} \dot{v}_{1}, l\right) \cdot h_{K_{1}, \delta}$ and $\left(\dot{u} \dot{v}_{2}, l^{\prime}\right) \cdot h_{K_{2}, \delta}$ are not in the same $G$ orbit. Thus $(g, g)\left(\dot{u} \dot{v}, l_{1}\right) \cdot h_{K, \delta}=\left(\dot{u} \dot{v}, l_{2}\right) \cdot h_{K, \delta}$ for some $v \in W_{\delta\left(I_{2}\right)}, K \subset I_{1}(J, u v, \delta)$ and $l_{1}, l_{2} \in$ $L_{I_{2}} \cap B$. By proposition 1.12, $g \in P_{I_{2}(K, u v, \delta)}$. Since $I_{2}(K, u v, \delta) \subset I_{2}(J, u v, \delta) \subset I_{2}$, we have that $g \in P_{I_{2}}$. By lemma $1.9, X_{u}^{(J, w, \delta)} \cong G \times_{P_{I_{2}}}\left(\left(P_{I_{2}}\right)_{d i a g} \cdot L_{u}^{(J, w, \delta)}\right)$. Part (2) is proved. 
For part (3), it is easy to see that $\left(P_{I_{2}(J, u, \delta)}\right)_{d i a g} \cdot L_{u}^{(J, w, \delta)} \subset(B \times B) \cdot L_{u}^{(J, w, \delta)}$. On the other hand,

$$
\begin{aligned}
(B \times B) \cdot L_{u}^{(J, w, \delta)} & =\left(U_{P_{I_{2}}}, U_{P_{I_{2}}}\right)\left(L_{I_{2}}\right)_{\text {diag }}\left(\sqcup_{v \in W_{\delta\left(I_{2}\right)}} \sqcup_{K \subset I_{2}(J, u v, \delta)}(\dot{u} \dot{v}, B) \cdot h_{K, \delta}\right) \\
& =\left(L_{I_{2}}\right)_{d i a g}\left(U_{P_{I_{2}}}, U_{P_{I_{2}}}\right)\left(\sqcup_{v \in W_{\delta\left(I_{2}\right)}} \sqcup_{K \subset I_{2}(J, u v, \delta)}(\dot{u} \dot{v}, B) \cdot h_{K, \delta}\right) .
\end{aligned}
$$

By proposition 1.12,

$$
\begin{aligned}
\left(U_{P_{I_{2}}}, U_{P_{I_{2}}}\right)(\dot{u} \dot{v}, B) \cdot h_{K, \delta} & =(B \times B)(\dot{u} \dot{v}, 1) \cdot h_{K, \delta} \\
& \subset\left(P_{I_{2}(K, u v, \delta)}\right)_{d i a g} \cdot\left(L_{I_{2}(K, u v, \delta)}, L_{I_{2}(K, u v, \delta)}\right)(\dot{u} \dot{v}, 1) h_{K, \delta} .
\end{aligned}
$$

We have showed that $I_{2}(K, u v, \delta) \subset I_{2}$. Hence

$$
\left(U_{P_{I_{2}}}, U_{P_{I_{2}}}\right)(\dot{u} \dot{v}, B) \cdot h_{K, \delta} \subset\left(P_{I_{2}(J, u, \delta)}\right)_{d i a g} \cdot L_{u}^{(J, w, \delta)} .
$$

Therefore, $\left(P_{I_{2}}\right)_{\text {diag }} \cdot L_{u}^{(J, w, \delta)}=(B \times B) \cdot L_{u}^{(J, w, \delta)}$.

Consider the morphism $\pi:\left(U \cap \dot{w}_{0}^{I_{2}} \dot{u} \dot{w}_{0} U^{-}\right) \times L_{u}^{(J, w, \delta)} \rightarrow(B \times B) \cdot L_{u}^{(J, w, \delta)}$ defined by $\pi(b, l)=(b, 1) \cdot l$ for $b \in U \cap \dot{w}_{0}^{I_{2}} \dot{u} \dot{w}_{0} U^{-}$and $l \in L_{u}^{(J, w, \delta)}$. By the similar argument as we did in proposition 1.10 , we can show that $\pi$ is an isomorphism.

Corollary 5.6. We keep the notation of proposition 5.5. If moreover, $I_{2}=\varnothing$, then $X_{u}^{(J, w, \delta)}$ admits a cellular decomposition.

Proof. If $I_{2}=\varnothing$, then $L_{u}^{(J, w, \delta)}$ is an affine space. Thus $X_{u}^{(J, w, \delta)}$ is isomorphic to $G \times B C$, where $C=(B \times B) \cdot L_{u}^{(J, w, \delta)}$. By part (3) of proposition 5.5, $C$ is an affine space. It is easy to see that $B$ acts linearly on $C$. Therefore $X_{u}^{(J, w, \delta)}$ is a vector bundle over $\mathcal{B}$. Note that $\mathcal{B}$ admits a cellular decomposition. By a well-known result (see Q, $[\mathrm{Su}]$ or $[\mathrm{VS}]$ ), $X_{u}^{(J, w, \delta)}$ admits a cellular decomposition.

5.7. For $w_{1}, w_{2} \in W_{\delta}(J, w)$, we say $w_{2} \leqslant w_{1}$ if there exists $w_{1}=x_{0}, x_{1}, \cdots, x_{n}=$

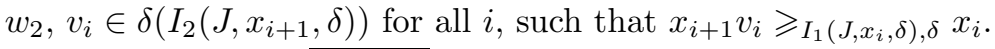

By theorem 4.5, $\overline{X_{u_{1}}^{(J, w, \delta)}} \cap X_{u_{2}}^{(J, w, \delta)}=\varnothing$ if $u_{2} \Varangle^{\prime} u_{1}$. Hence if $\leqslant$ is a partial order on $W_{\delta}(J, w)$, then $\overline{Z_{J, \delta}^{w}}=\sqcup_{u \in W_{\delta}(J, w)} X_{u}^{(J, w, \delta)}$ is an $\alpha$-partition. We will show that $\leqslant^{\prime}$ is a partial order if $\overline{Z_{J, \delta}^{w}}$ contains finitely many $G$-orbits.

Lemma 5.8. Let $J \subset I, u \in W, w \in W^{J}$ and $v \in W_{J}$. Assume that $u w v=w^{\prime} v^{\prime}$ for some $w^{\prime} \in W^{J}$ and $v^{\prime} \in W_{J}$. If $l(u w v)=l(w v)-l(u)$, then $w^{\prime} \leqslant w$. If moreover, $w^{\prime}=w$, then $\operatorname{Ad}\left(w^{-1}\right) \operatorname{supp}(u) \subset J$.

Proof. If $u=s_{i}$ for some $i \in J$ and $l\left(s_{j} w v\right)=l(w v)-1$, then either $s_{i} w<w$ and $s_{i} w \in W^{J}$ or $s_{i} w=w s_{j}$ for some $j \in J$. It is easy to check that the statement holds in both cases.

The general case can be proved by induction on $l(u)$.

Lemma 5.9. If $w_{1}, w_{2} \in W_{\delta}(J, w)$ with $w_{1} \leqslant{ }^{\prime} w_{2}$ and $w_{2} \leqslant{ }^{\prime} w_{1}$ and $I_{2}\left(J, w_{1}, \delta\right)=$ $I_{2}\left(J, w_{2}, \delta\right)=\varnothing$, then $w_{1}=w_{2}$.

Proof. We will prove the case: if $w_{1} \geqslant_{I_{1}\left(J, w_{2}, \delta\right), \delta} w_{2}, w_{2} \geqslant_{I_{1}\left(J, w_{1}, \delta\right), \delta} w_{1}$ and $I_{2}\left(J, w_{1}, \delta\right)=\varnothing$, then $w_{1}=w_{2}$. The general case can be proved in the same way. 
We argue by induction on $|J|$. Since $l\left(w_{1}\right) \geqslant l\left(w_{2}\right)$ and $l\left(w_{2}\right) \geqslant l\left(w_{1}\right)$, we have that $l\left(w_{1}\right)=l\left(w_{2}\right)$. Thus $w_{1}=u_{2}^{-1} w_{2} \delta\left(u_{2}\right)$ and $w_{2}=u_{1}^{-1} w_{1} \delta\left(u_{1}\right)$ for some $u_{1} \in W_{I_{1}\left(J, w_{1}, \delta\right)}$ and $u_{2} \in W_{I_{1}\left(J, w_{2}, \delta\right)}$. By induction hypothesis, it suffices to prove the case when $J=\operatorname{supp}\left(u_{1}\right) \cup \operatorname{supp}\left(u_{2}\right)$.

We have that $w_{1}=w_{1}^{\prime} \delta\left(v_{1}\right)$ and $w_{2}=w_{2}^{\prime} \delta\left(v_{2}\right)$ for some $w_{1}^{\prime}, w_{2}^{\prime} \in W^{\delta(J)}$ and $v_{1}, v_{2} \in W_{J}$. Note that $w_{1}^{\prime} \delta\left(v_{1}\right)=u_{2}^{-1} w_{2}^{\prime} \delta\left(v_{2} u_{2}\right)$ and $l\left(u_{2}^{-1} w_{2}^{\prime} \delta\left(v_{2} u_{2}\right)\right)=$ $l\left(w_{2}^{\prime} \delta\left(v_{2} u_{2}\right)\right)-l\left(u_{2}\right)$. By lemma 5.8, $w_{1}^{\prime} \leqslant w_{2}^{\prime}$. Similarly $w_{2}^{\prime} \leqslant w_{1}^{\prime}$. Therefore $w_{1}^{\prime}=w_{2}^{\prime}$. By 5.8, $\operatorname{Ad}\left(w_{2}^{\prime}\right)^{-1} \operatorname{supp}\left(u_{2}\right) \subset \delta(J)$ and $\operatorname{Ad}\left(w_{1}^{\prime}\right)^{-1} \operatorname{supp}\left(u_{1}\right) \subset \delta(J)$. Therefore $\operatorname{Ad}\left(w_{1}^{\prime}\right)^{-1} J \subset \delta(J)$. Hence $\operatorname{Ad}\left(w_{1}\right)^{-1} \Phi_{J}=\Phi_{\delta(J)}$. Since $I_{2}\left(J, w_{1}, \delta\right)=\varnothing$, we have that $J=\varnothing$. Therefore $w_{1} \geqslant w_{2}$ and $w_{2} \geqslant w_{1}$. Thus $w_{1}=w_{2}$. The case is proved.

As a summary, we have the following result.

Theorem 5.10. If $\overline{Z_{J, \delta}^{w}}$ contains only finitely many $G$-orbits, then it has a cellular decomposition.

Proof. If $\overline{Z_{J, \delta}^{w}}$ contains only finitely many $G$-orbits, then $I_{2}(J, u, \delta)=\varnothing$ for $u \geqslant_{J, \delta}$ $w$. In this case, $W_{\delta}(J, w)=\left\{u \in W \mid u \geqslant_{J, \delta} w\right\}$. We have that

$$
\overline{Z_{J, \delta}^{w}}=\sqcup_{u \in W_{\delta}(J, \delta)} X_{u}^{(J, w, \delta)} .
$$

By section 5.7 and lemma 5.9, the partition is an $\alpha$-partition. Thus by corollary $5.6, \overline{Z_{J, \delta}^{w}}$ has a cellular decomposition.

\section{ACKNOWLEDGEMENTS}

We thank George Lusztig for suggesting the problem and for many helpful discussions. We thank T. A. Springer and David Vogan for some useful comments. We also thank Jiang-hua Lu for pointing out a mistake in the previous version and suggesting the reference $[\mathrm{SL}$.

\section{REFERENCES}

[DP] C. De Concini and C.Procesi, Complete symmetric varieties, Invariant theory (Montecatini 1982), Lect. Notes Math., vol. 996, Springer, 1983, pp. 1-44. MR0718125 (85e:14070)

$[\mathrm{H}] \quad \mathrm{X} . \mathrm{He}$, Unipotent variety in the group compactification, Adv. in Math., 203 (2006), 109-131. MR2231043

[L1] G. Lusztig, Total positivity in reductive groups, Lie Theory and Geometry: in honor of Bertram Kostant, Progress in Math. 123 (1994), 531-568. MR1327548 (96m:20071)

[L2] G. Lusztig, Hecke algebras with unequal parameters, CRM Monograph Series, 18, American Mathematical Society, Providence, RI, 2003. MR1974442 (2004k:20011)

[L3] G. Lusztig, Parabolic character sheaves I, Moscow Math.J 4 (2004), 153-179. MR2074987 (2006d:20091a)

[L4] G. Lusztig, Parabolic character sheaves II, Moscow Math.J 4 (2004), 869-896. MR 2124170 (2006d:20091b)

[Q] D. Quillen, Projective modules over polynomial rings, Invent. Math. 36 (1976), 167-171. MR0427303 (55:337)

[S] T. A. Springer, Intersection cohomology of $B \times B$-orbits closures in group compactifications, J. Alg. 258 (2002), 71-111. MR1958898 (2004a:14025)

[SL] P. Slodowy, Simple singularities and simple algebraic groups, Lecture Notes in Mathematics, 815, Springer, Berlin, 1980. MR0584445 (82g:14037) 
[Su] A. A. Suslin, Projective modules over polynomial rings are free (Russian), Dokl. Akad. Nauk SSSR 229 (1976), no. 5, 1063-1066. MR0469905 (57:9685)

[VS] L. N. Vaserstein and A. A. Suslin, Serre's problem on projective modules over polynomial rings, and algebraic K-theory (Russian), Izv. Akad. Nauk SSSR Ser. Mat. 40 (1976), no. 5, 993-1054, 1199. MR0447245 (56:5560)

Department of Mathematics, M.I.T., Cambridge, Massachusetts 02139

E-mail address: xuhua@mit.edu, hugo@math.mit.edu

Current address: Department of Mathematics, Stony Brook University, Stony Brook, New York 11794

E-mail address: hugo@math.sunysb.edu 